\title{
LA-UR-17-28269
}

Approved for public release; distribution is unlimited.

Title: $\quad$ Standardized Definitions for Code Verification Test Problems

Author(s): $\quad$ Doebling, Scott William

Intended for: $\quad$ Report

Issued: 
Disclaimer:

Los Alamos National Laboratory, an affirmative action/equal opportunity employer, is operated by the Los Alamos National Security, LLC for the National Nuclear Security Administration of the U.S. Department of Energy under contract DE-AC52-06NA25396. By approving this article, the publisher recognizes that the U.S. Government retains nonexclusive, royalty-free license to publish or reproduce the published form of this contribution, or to allow others to do so, for U.S. Government purposes. Los Alamos National Laboratory requests that the publisher identify this article as work performed under the auspices of the U.S. Department of Energy. Los Alamos National Laboratory strongly supports academic freedom and a researcher's right to publish; as an institution, however, the Laboratory does not endorse the viewpoint of a publication or guarantee its technical correctness. 


\section{Standardized Definitions for Code Verification Test Problems}

Release 1.0

LANL Physics Verification Team, LA-UR-17-28269 

CONTENTS

1 Abstract 1

2 The Noh Problem $\quad 3$

$\begin{array}{lll}3 & \text { The Sedov Problem } & 7\end{array}$

4 Cog-8 Problem 11

5 The Escape of HE Products (EHEP) Problem 15

6 Guderley Problem $\quad 19$

7 1D JWL Riemann Problems $\quad 23$

8 Kenamond Problem \#1 27

9 Kenamond Problem \#2 $\quad 31$

10 Kenamond Problem \#3 $\quad 37$

11 Kidder Problem $\quad 43$

12 1D Plasma Diffusion Problems $\quad 47$

13 1D Riemann Problems $\quad 51$

14 Numerical Setup for 1D Riemann Problems $\quad 61$

15 Mader Problem $\quad 63$

16 The Blake Problem $\quad 69$

17 The Hunter Problem $\quad 73$

$\begin{array}{ll}\text { Bibliography } & 77\end{array}$ 
This document contains standardized definitions for several commonly used code verification test problems. These definitions are intended to contain sufficient information to set up the test problem in a computational physics code. These definitions are intended to be used in conjunction with exact solutions to these problems generated using ExactPack, www.github.com/lanl/exactpack

To provide feedback, contact Scott Doebling, doebling@lanl.gov 


\subsection{Description}

A numerical implementation of the Noh Problem involves a finite-domain restriction of the mathematically ideal, infinite domain, spherically symmetric, infinite- strength shock impinging on a rigid wall. It consists of an inviscid, non-heat conducting, compressible, polytropic gas, initialized with a uniform, spherically radially inward velocity, $u_{R, 0}$. This problem tests a code's ability to convert kinetic energy into internal energy.

The Noh problem was first published by William F. Noh in 1987 [Noh]. See also [Kamm].
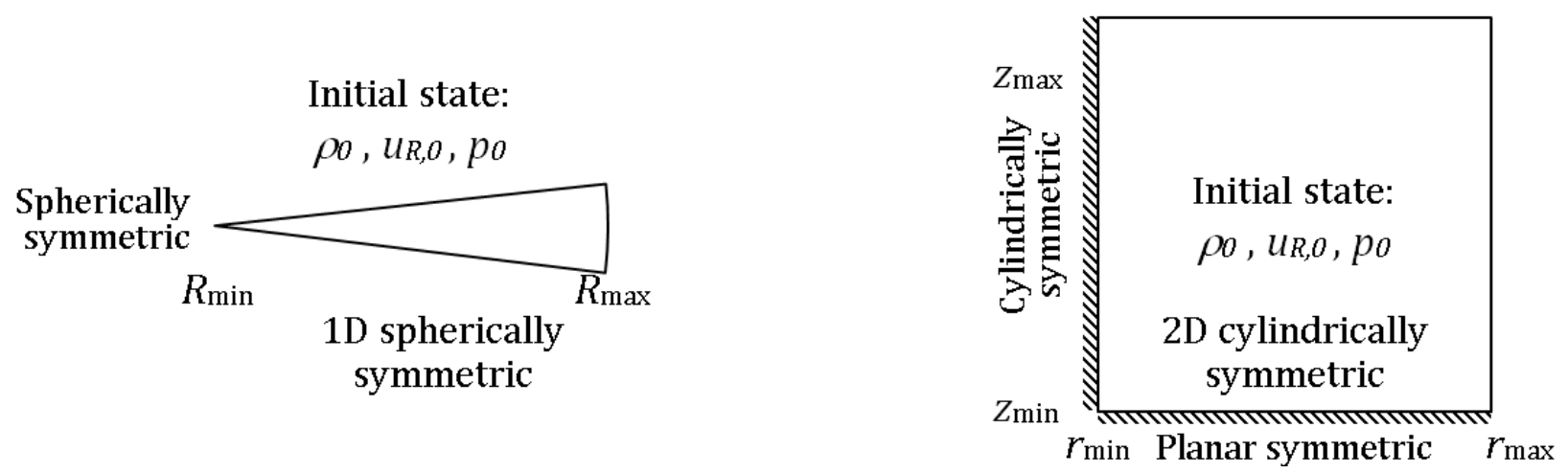

Fig. 2.1: Initial configuration of the 1D and 2D Spherical Noh Problem.

\subsection{Solution}

Table 2.1: Problem solution at $t_{\text {fin }}=0.6$

\begin{tabular}{|l|l|l|l|l|}
\hline$r$ & $\rho$ & $u_{R}$ & $p$ & $e$ \\
\hline$[\mathrm{cm}]$ & {$\left[\mathrm{g} / \mathrm{cm}^{3}\right]$} & {$[\mathrm{cm} / \mathrm{s}]$} & {$\left[\mathrm{dyn} / \mathrm{cm}^{2}\right]$} & {$[\mathrm{erg} / \mathrm{g}]$} \\
\hline$R<R_{s}=0.2$ & 64.0 & 0.0 & $21 \frac{1}{3}$ & $1 / 2$ \\
\hline$R>R_{s}=0.2$ & {$[1+(0.6 / R)]^{2}$} & -1.0 & $(2 / 3) \rho \times 10^{-12}$ & $1 \times 10^{-12}$ \\
\hline
\end{tabular}




\subsection{Problem Parameters}

Table 2.2: Parameters for the Noh problem.

\begin{tabular}{|l|l|l|l|l|l|l|}
\hline & $t_{\text {fin }}$ & $\gamma$ & $\rho_{0}$ & $u_{0}$ & $p_{0}$ & $E_{0}$ \\
\hline & {$[\mathrm{s}]$} & {$[-]$} & {$\left[\mathrm{g} / \mathrm{cm}^{3}\right]$} & {$[\mathrm{cm} / \mathrm{s}]$} & {$\left[\mathrm{dyn} / \mathrm{cm}^{2}\right]$} & {$[\mathrm{erg}]$} \\
\hline 1D & 0.6 & $5 / 3$ & 1.0 & $u_{R, 0}=-1.0$ & $(2 / 3) \times 10^{-12}$ & $1 \times 10^{-12}$ \\
\hline 2D & 0.6 & $5 / 3$ & 1.0 & $u_{r, 0}=-r / R$, & $(2 / 3) \times 10^{-12}$ & $1 \times 10^{-12}$ \\
\hline & & & & $u_{z, 0}=-z / R$ & & \\
\hline 3D & 0.6 & $5 / 3$ & 1.0 & $u_{x, 0}=-x / R$, & $(2 / 3) \times 10^{-12}$ & $1 \times 10^{-12}$ \\
\hline & & & & $u_{y, 0}=-y / R$, & & \\
\hline & & & & $u_{z, 0}=-z / R$ & & \\
\hline
\end{tabular}

\subsection{Initial Conditions}

Uniform and constant material density, pressure, and spherically radial velocity. States are related through the polytropic (ideal gas) EOS: $p=(\gamma-1) \rho e$, where, $\gamma=5 / 3$ is the (constant) ratio of specific heats.

\subsection{Boundary Conditions}

The problem is to be run so that any spurious waves generated by boundary conditions do not affect the solution on the Comparison Domain, defined below. Possible boundary conditions are:

Inner: $R_{\min }, r_{\min }, z_{\min }:$ Symmetric

Outer: $R_{\max }, r_{\max }, z_{\max }$ : Constant in time

\subsection{Mesh}

Region Boundaries ${ }^{1}$ :

1D: $R_{\min }=0.0, R_{\max }=1.2 \mathrm{~cm}$

2D: $(r, z)_{\min }=0.0,(r, z)_{\max }=1.2 \mathrm{~cm}$

3D: $(x, y, z)_{\min }=0.0,(x, y, z)_{\max }=1.2 \mathrm{~cm}$

Mesh Resolutions:

1D spherical: $N_{R}=24,48,96,192,384 ; \Delta_{R}=0.5,0.025,0.0125,0.00625,0.003125$

2D cylindrical: $N_{r}=N_{z}=24,48,96,192,384 ; \Delta_{r}=\Delta_{z}=0.5,0.025,0.0125,0.00625,0.003125$

3D Cartesian $N_{x}=N_{y}=N_{z}=24,48,96,192 ; \Delta_{x}=\Delta_{y}=\Delta_{z}=0.5,0.025,0.0125,0.00625$

\subsection{Output}

In ASCII comma- or space-delimited format, ${ }^{2}$ to include:

\footnotetext{
${ }^{1}$ Here, $R=$ spherical radial coordinate, with $R=r^{2}+z^{2}$, where $r=$ cylindrical radial coordinate.

${ }^{2}$ Sample output available upon request.
} 
1. Values of density, velocity, pressure, SIE as a function of position at $t_{0}$ and $t_{\text {fin }}$.

2. Entire mesh total energy, kinetic energy, internal energy as a function of time.

\subsection{Comparison Domain}

1D: $R \in[0,0.5]$

2D: $(r, z) \in[0,0.5] \times[0,0.5]$

3D: $(x, y, x) \in[0,0.5] \times[0,0.5] \times[0,0.5]$

2.9 Results at $t_{\text {fin }}=0.6 \mathbf{s}$
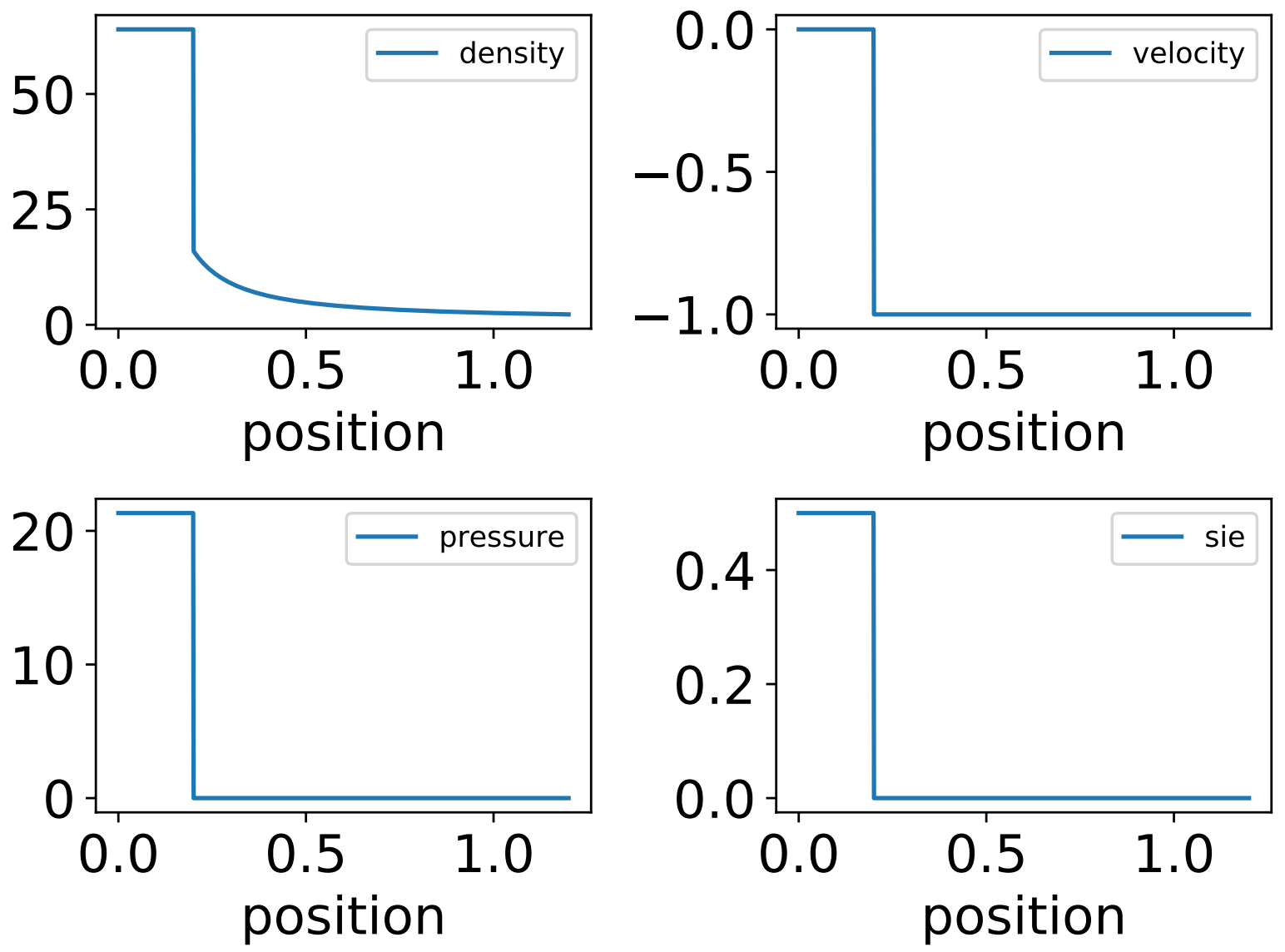


\subsection{Description}

The Sedov Problem is a mathematical idealization of a shock generated via an explosion. It consists of spherically symmetric flow of an inviscid, non-heat conducting, compressible, polytropic gas, driven by a single zone with nontrivial initial energy $[\mathrm{Kamm}]$.

This problem tests a code's ability to convert internal energy into kinetic energy and has a quasi-analytic, self-similar solution that requires one numerical quadrature.

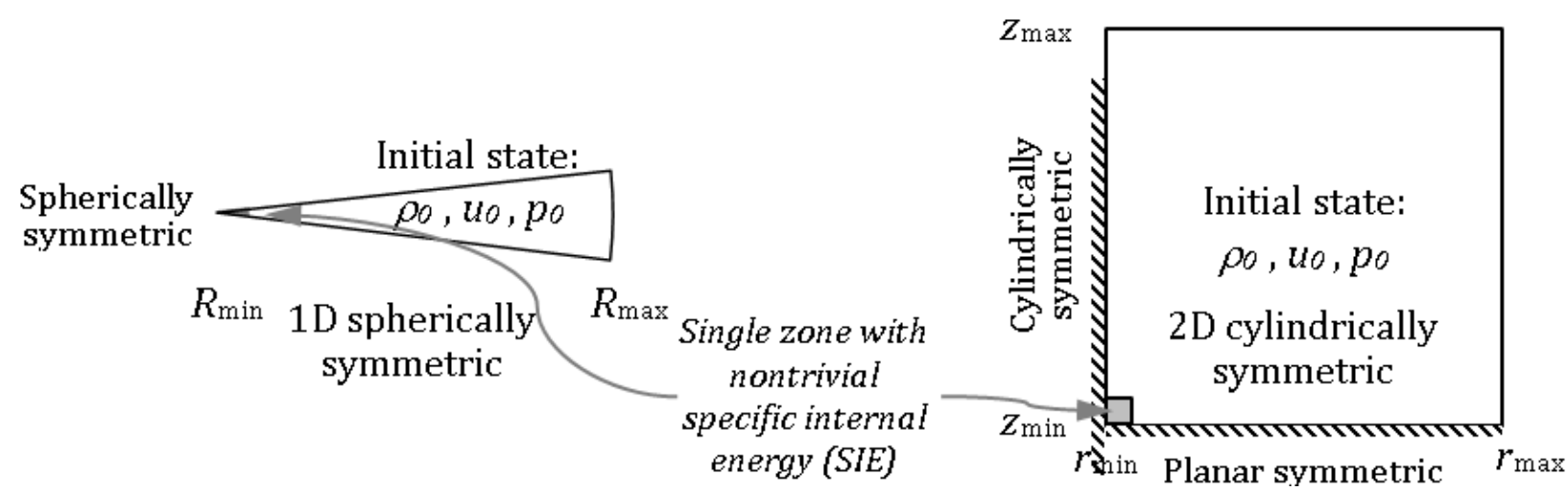

Fig. 3.1: Initial Configuration of the 1D and 2D Spherical Sedov problem.

\subsection{Problem Parameters}

Table 3.1: Parameters for the Sedov problem.

\begin{tabular}{|c|l|l|l|l|l|}
\hline$t_{\text {fin }}$ & $\gamma$ & $\rho_{0}$ & $u_{0}$ & $p_{0}$ & Internal Energy \\
\hline$[\mathrm{s}]$ & {$[-]$} & {$\left[\mathrm{g} / \mathrm{cm}^{3}\right]$} & {$[\mathrm{cm} / \mathrm{s}]$} & {$\left[\mathrm{dyn} / \mathrm{cm}^{2}\right]$} & {$[\mathrm{erg}]$} \\
\hline 1.0 & $7 / 5$ & 1.0 & 0.0 & $(2 / 5) \times 10^{-12}$ & 1D: 0.851072 \\
\hline & & & & & 2D: 0.425536 \\
\hline & & & & & 3D: 0.106384 \\
\hline
\end{tabular}




\subsection{Initial Conditions}

Uniform and constant material density and velocity. Specific Internal Energy (SIE) in the single energetic zone as shown in the SIE table below, elsewhere $10^{-12} \mathrm{erg} / \mathrm{g}$, so that pressure and total energy equal values in the Paramters. States are related through the polytropic (ideal gas) EOS: $p=(\gamma-1) \rho e$, where, $\gamma=7 / 5$ is the (constant) ratio of specific heats.

Table 3.2: SIE [erg/g] in first zone gives the internal energy in the Parameters table.

\begin{tabular}{|l|l|l|l|l|l|}
\hline $\mathrm{D}$ & 24 & 48 & 96 & 192 & 384 \\
\hline 1D & $1.6254278 \times 10^{3}$ & $1.3003422 \times 10^{4}$ & $1.0402738 \times 10^{5}$ & $8.3221903 \times 10^{5}$ & $6.6577522 \times 10^{6}$ \\
\hline 2D & $1.0836185 \times 10^{3}$ & $8.6689482 \times 10^{3}$ & $6.9351586 \times 10^{4}$ & $5.5481269 \times 10^{5}$ & $4.4385015 \times 10^{6}$ \\
\hline 3D & $8.5107200 \times 10^{2}$ & $6.8085760 \times 10^{3}$ & $5.4468608 \times 10^{4}$ & $4.3574886 \times 10^{5}$ & \\
\hline
\end{tabular}

\subsection{Boundary Conditions}

Inner: $R_{\min }, r_{\min }, z_{\min }:$ Symmetric

Outer: $R_{\max }, r_{\max }, z_{\max }:$ Constant in time

\subsection{Mesh}

Region Boundaries ${ }^{1}$ :

$R_{\min }=0.0, R_{\max }=1.2 \mathrm{~cm} ;$ in $1 \mathrm{D}$

$(r, z)_{\min }=0.0,(r, z)_{\max }=1.2 \mathrm{~cm} ;$ in $2 \mathrm{D}$,

$(x, y, z)_{\min }=0.0,(x, y, z)_{\max }=1.2 \mathrm{~cm} ;$ in 3D

Mesh Resolutions:

1D spherical: $N_{R}=24,48,96,192,384 ; \Delta_{R}=0.5,0.025,0.0125,0.00625,0.003125$

2D cylindrical: $N_{r}=N_{z}=24,48,96,192,384 ; \Delta_{r}=\Delta_{z}=0.5,0.025,0.0125,0.00625,0.003125$

3D Cartesian $N_{x}=N_{y}=N_{z}=24,48,96,192 ; \Delta_{x}=\Delta_{y}=\Delta_{z}=0.5,0.025,0.0125,0.00625$

\subsection{Output}

In ASCII comma- or space-delimited format, ${ }^{2}$ to include:

1. Values of density, velocity, pressure, SIE as a function of position at $t_{0}$ and $t_{\text {fin }}$.

2. Entire mesh total energy, kinetic energy, internal energy as a function of time.

\footnotetext{
${ }^{1}$ Here, $R=$ spherical radial coordinate, with $R=r^{2}+z^{2}$, where $r=$ cylindrical radial coordinate.

${ }^{2}$ Sample output available upon request.
} 


\subsection{Comparison Domain}

1D: $R \in[0,1.2]$

2D: $(r, z) \in[0,1.2] \times[0,1.2]$

3D: $(x, y, z) \in[0,1.2] \times[0,1.2] \times[0,1.2]$

\subsection{Results at $t_{\text {fin }}=1.0$}
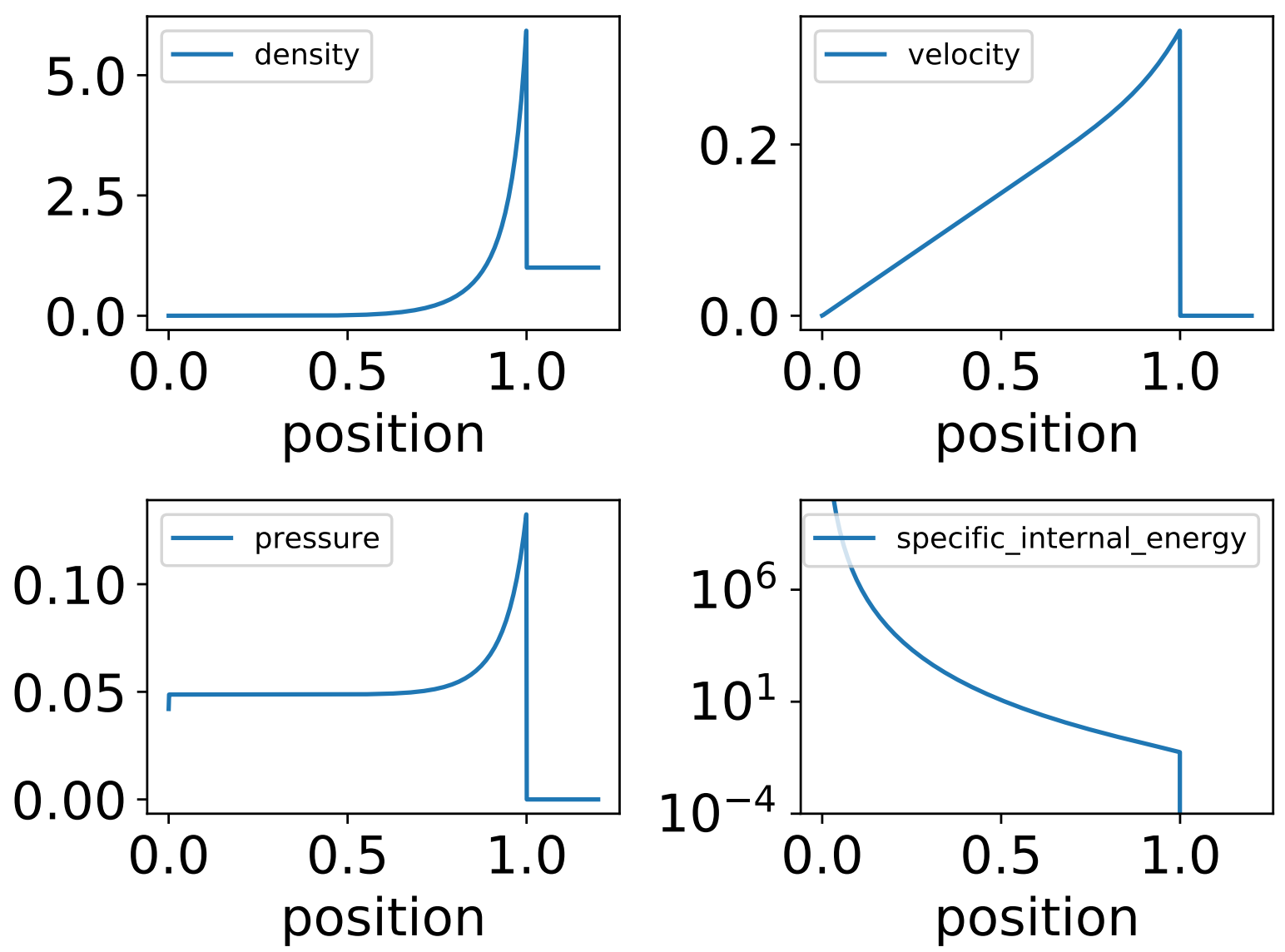


\section{COG-8 PROBLEM}

\subsection{Description}

The Cylindrical Cog-8 Problem is a cylindrically symmetric configuration that couples the adiabatic expansion of a compressible gas with radiation-diffusion having a specific heat flux. The initial state of the inviscid, polytropic gas must be assigned numerically. This problem tests a code's ability to resolve a simple, smooth multiphysics scenario. This problem was originally given as one of a group of 22 formal similiarity solutions of th Euler compressible flow equations which Cogesshall derived using Lie group theory [Coggeshall]. The particular solution corresponding to the Cog- 8 problems was analyzed in detail ${ }^{1}$ in the paper by Hendon and Ramsey [Hendon]. See also [Kamm] .
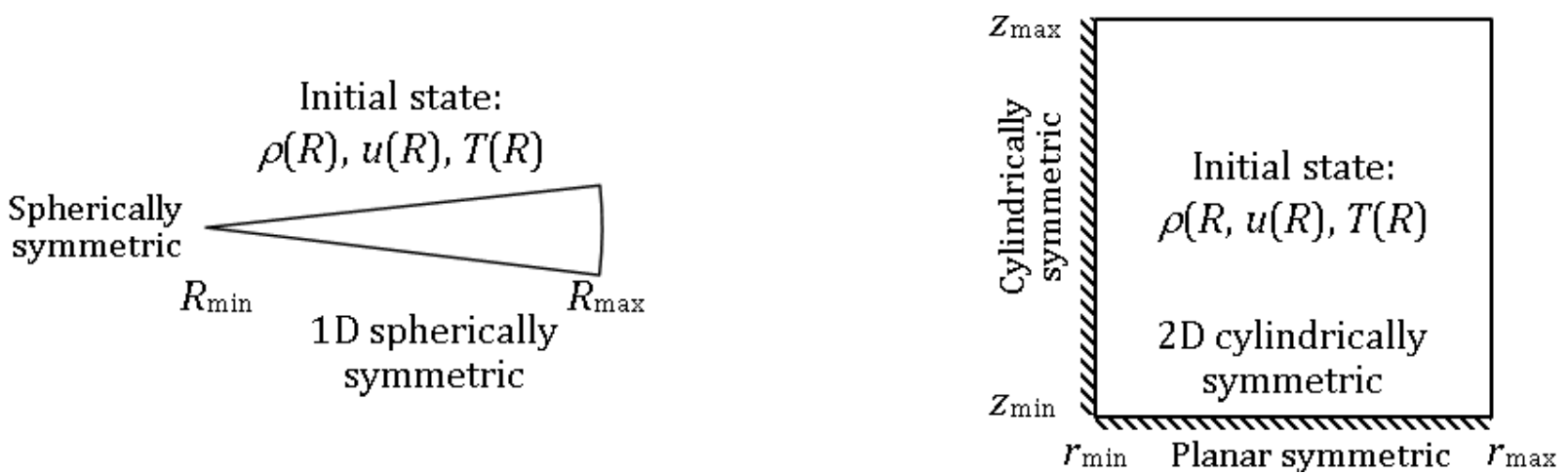

Fig. 4.1: Initial configuration of the Cog-8 Problem

\subsection{Solution}

Of Coggeshall's 22 similarity soltuions, the Cog-8 problem corresonds to a formal solution invariant under scale transormations on $\rho$ and spatial scale transformations, along with radiation diffusion (or nonlinear heat conduction). The solution contains five unspecified parameters: The gas constants, $\gamma$ and, either $C_{\mathrm{V}}$ or $\Gamma=C_{\mathrm{V}}(\gamma-1)$, and three arbitrary constants, $\kappa_{0}, A$ and $B$, in Coggeshalls notation or $\alpha=A$ and $\beta=B-3$, in Hendon and Ramsey's notation. The latter three parameters appear in the parameterization of the Rosseland mean opacity (or nonlinear heat conduction), chosen to be of exponential form,

$$
\kappa(\rho, T)=\kappa_{0} \rho^{A} T^{B}
$$

\footnotetext{
${ }^{1}$ Hendon and Ramsey show that, in general, a delta-function heat source term must be included to reproduce the complete solution, but not for the case considered here.
} 
in Coggeshall's notation, or,

$$
\kappa(\rho, T)=\kappa_{0} \rho^{\alpha} T^{\beta+3}
$$

in Hendon and Ramsey's notation.

For a polytropic gas, with $p=(\gamma-1) \rho e=C_{\mathrm{V}}(\gamma-1) \rho T$, and temperature-dependent Rosseland mean opacity,

$$
\kappa=\kappa_{0} \rho^{-1} T^{3},
$$

the Cog-8 problem has the closed-form solution ${ }^{2}$ given in the Solution table below.

Table 4.1: Solution to the Cylindrical Cog-8 problem.

\begin{tabular}{|l|l|}
\hline Physical Field & Analytic Solution \\
\hline$\rho\left[\mathrm{g} / \mathrm{cm}^{3}\right]$ & $\rho_{0} t^{-2}$ \\
\hline$u[\mathrm{~cm} / \mathrm{s}]$ & $r / t$ \\
\hline$e[\mathrm{erg} / \mathrm{g}]$ & $T_{0} t^{-4 / 3}$ \\
\hline
\end{tabular}

\subsection{Problem Parameters}

Parameters for the problem considered here are listed in the Parameters table.

Table 4.2: Parameters for the Cog-8 problem.

\begin{tabular}{|l|l|l|l|l|l|l|}
\hline$t_{\text {init }}$ & $t_{\text {fin }}$ & $\gamma$ & $C_{\mathrm{V}}$ & $\kappa_{0}$ & $\alpha$ & $\beta$ \\
\hline$[\mathrm{s}]$ & {$[\mathrm{s}]$} & {$[-]$} & {$[\mathrm{erg} /(\mathrm{g} \mathrm{K})]$} & {$\left[\mathrm{cm}^{2} / \mathrm{g}\right]$} & {$[-]$} & {$[-]$} \\
\hline 1.0 & 2.0 & $5 / 3$ & $1.0 \times 10^{7}$ & 1.0 & 0 & 0 \\
\hline
\end{tabular}

\subsection{Initial Conditions}

Spatially dependent material state over the entire domain. The initial values $\rho_{0}$ and $T_{0}$ are found by substituting $t=1$ into the Solution table. They are given in the Initial values table below. Note that this table is redunant for setting up the problem. Setup only requires imposing the initial velocity, density and either the temperature or the specific internal energy (SIE). The SIE is determined by the temperature via $e=C_{\mathrm{V}} T$. The pressure is determined from the other two variables, since $p=(\gamma-1) \rho T$, but it was included in the table for completeness, and because it can be used as a consistency check on input decks at the beginning of a calculation.

Table 4.3: Initial values of the Cog-8

Problem

\begin{tabular}{|l|l|}
\hline Physical Field & Analytic Solution \\
\hline$\rho\left[\mathrm{g} / \mathrm{cm}^{3}\right]$ & 1.0 \\
\hline$T_{0}[\mathrm{~K}]$ & $1.16045 \times 10^{4}$ \\
\hline$e[\mathrm{erg} / \mathrm{g}]$ & $1.16045 \times 10^{11}$ \\
\hline$u[\mathrm{~cm} / \mathrm{s}]$ & $r$ \\
\hline$p[\mathrm{Mbar}]$ & $7.73633 \times 10^{3}$ \\
\hline
\end{tabular}

\footnotetext{
${ }^{2}$ See section III.A of the report by Hendon and Ramsey. This configuration corresponds to the parameters $\kappa=1, \gamma=5 / 3, \mathrm{~A}=0$, and $\beta=-3$ (or $\kappa=1, \gamma=5 / 3, A=0$, and $B=0$ in Coggeshall's notation).
} 


\subsection{Boundary Conditions}

The problem is to be run so that any spurious waves generated by boundary conditions do not affect the solution on the Comparison Domain, defined below. Possible boundary conditions are:

Inner: $r_{\min }, z_{\min }:$ Symmetric

Outer: $r_{\max }, z_{\max }:$ Constant in time

\subsection{Mesh}

Region Boundaries ${ }^{3}$ :

1D: $R_{\min }=0.0, R_{\max }=1.2 \mathrm{~cm}$

2D: $(r, z)_{\min }=0.0,(r, z)_{\max }=1.2 \mathrm{~cm}$

Mesh Resolutions:

1D spherical: $N_{r}=24,48,96,192,384 ; \Delta_{R}=0.5,0.025,0.0125,0.00625,0.003125$

2D cylindrical: $N_{r}=N_{z}=24,48,96,192,384 ; \Delta_{r}=\Delta_{z}=0.5,0.025,0.0125,0.00625,0.003125$

\subsection{Output}

In ASCII comma- or space-delimited format, ${ }^{4}$ to include:

1. Values of density, velocity, pressure, SIE as a function of position at $t_{\text {init }}$ and $t_{\text {fin }}$.

2. Entire mesh total energy, kinetic energy, internal energy as a function of time.

\subsection{Comparison Domain}

1D: $R \in[0,0.5]$

2D: $(r, z) \in[0,0.5] \times[0,0.5]$

\subsection{Results at $t_{\text {fin }}=0.6 \mathbf{s}$}

\footnotetext{
${ }^{3}$ Here, $R=$ spherical radial coordinate, with $R=r^{2}+z^{2}$, where $r=$ cylindrical radial coordinate.

${ }^{4}$ Sample output available upon request.
} 


\section{THE ESCAPE OF HE PRODUCTS (EHEP) PROBLEM}

\subsection{Description}

The escape of HE products (EHEP) problem involves a one-dimensional rod of HE extending from the origin for a length of $\tilde{x}$. To the left of the HE is a piston moving in the $+x$ direction with velocity $u_{p}$. To the right of the HE is a void.

At $t=0$, the detonation wave departs from the origin in the $+x$ direction, and the piston begins to move and isentropically compresses the reaction products. When the detonation wave reaches $\tilde{x}$ the HE has all been consumed, and the material begins to expand isentropically into the void region. At the same time, the arrival of the detonation wave at an interface with lesser impedence to the right causes a rarefaction wave to be propagated from $\tilde{x}$ in the $-x$ direction. A bit later, this rarefaction wave impacts the piston and reflects back into the material. Thus there are five distinct regions of behavior as the HE detonates and the reaction products spread.

The HE is assumed to be a polytropic ideal gas with adiabatic index $\gamma=3$, C-J detonation velocity $D$, and heat of reaction $q$. (The value $\gamma=3$ is required to enable the derivation of the exact solution.) The unreacted HE and the reacted $\mathrm{HE}$ are assumed to have the same material properties.

The EHEP problem was first published by Fickett and Rivard in 1974 [Fickett]. In 2002, [Dykema] published a derivation of the characteristics of the exact solution in $x$ - $t$ space. A complete description of the problem, the exact solution equations, and the solution algorithm is presented in [Doebling].






\subsection{Problem Parameters}

$$
\begin{aligned}
t_{\text {final }} & =5.0 \mu \mathrm{s} \\
\gamma & =3 \\
D & =0.85 \mathrm{~cm} / \mu \mathrm{s} \\
q & =0.04515625 \mathrm{~cm}^{2} / \mu \mathrm{s}^{2}
\end{aligned}
$$

\subsection{Mesh: 1D Cartesian}

For a Lagrangian code, the mesh should be defined as indicated with the number of zones $N_{x}$ defined over the initial HE region $[0, \tilde{x}]$. The void region need not be meshed. For an Eulerian or ALE code, the mesh should be entended from $\tilde{x}$ to $\hat{x}$ using the same $\mathrm{dx}$ values as in the HE region.

$$
\begin{aligned}
\tilde{x} & =1 \mathrm{~cm} \\
\hat{x} & =5.0 \mathrm{~cm} \\
N_{x} & =[50,100,200,400,800] \text { over interval }[0, \tilde{x}] \mathrm{cm} \\
\Delta x & =0.02,0.01,0.005,0.0025,0.00125
\end{aligned}
$$

\subsection{Initial conditions}

$$
\begin{aligned}
& p_{0}=0.0 \\
& \rho_{0}=1.6 \mathrm{~g} / \mathrm{cm}^{3} \\
& e_{0}=0.0
\end{aligned}
$$

\subsection{Boundary conditions}

The left $(-x)$ boundary is being pushed by the piston at velocity $u_{p}$. The right $(+x)$ boundary of the problem is free to expand.

$$
u_{p}=0.05 \mathrm{~cm} / \mu \mathrm{s}
$$

\subsection{Comparison Domain}

The comparison doman is $x \in[0.0,5.0] \mathrm{cm}$.

\subsection{Outputs}

Output should be provided in ASCII comma or space-delimited format, to include:

1. Values of density, velocity, pressure, and specific internal energy as a function of position at $t=0$ and $t_{\text {final }}$.

2. Entire mesh total energy, kinetic energy, internal energy as a function of time. 


\subsection{Exact Solution plots}

At time $t=5.0 \mu \mathrm{sec}$
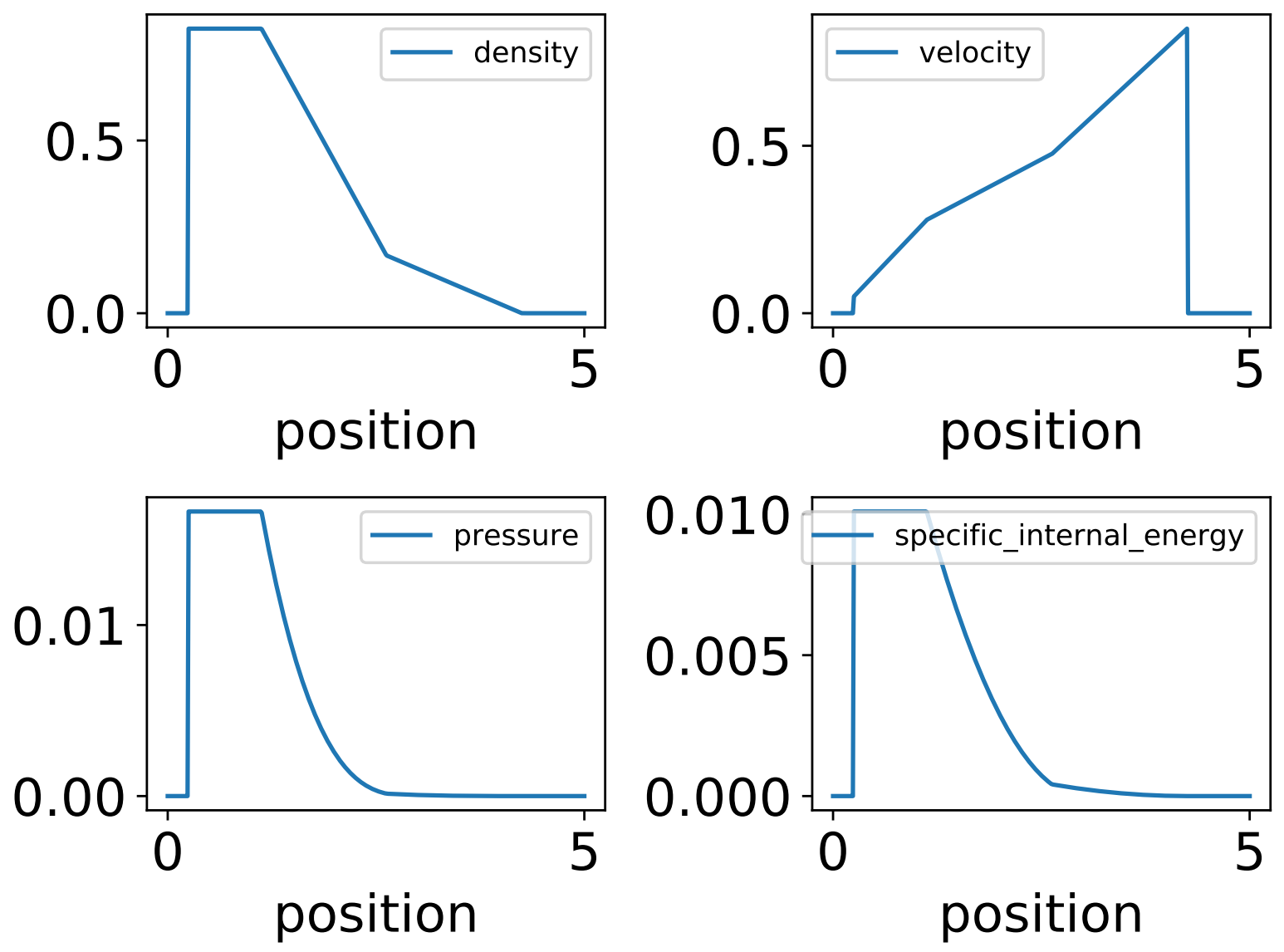


\subsection{Description}

The Guderley Problem consists of a spherically symmetric, shocked flow that converges onto the origin, bounces and reflects outward into the oncoming flow. The initial state of the inviscid, non-heat conducting polytropic gas is uniform out to a specified radius, but spatially dependent beyond and must be evaluated numerically. This problem tests a code's ability to resolve spherically converging reflected shocked flow.

The Guderley Problem was first published by Guderley in 1942 [Guderley]. See also [Kamm].
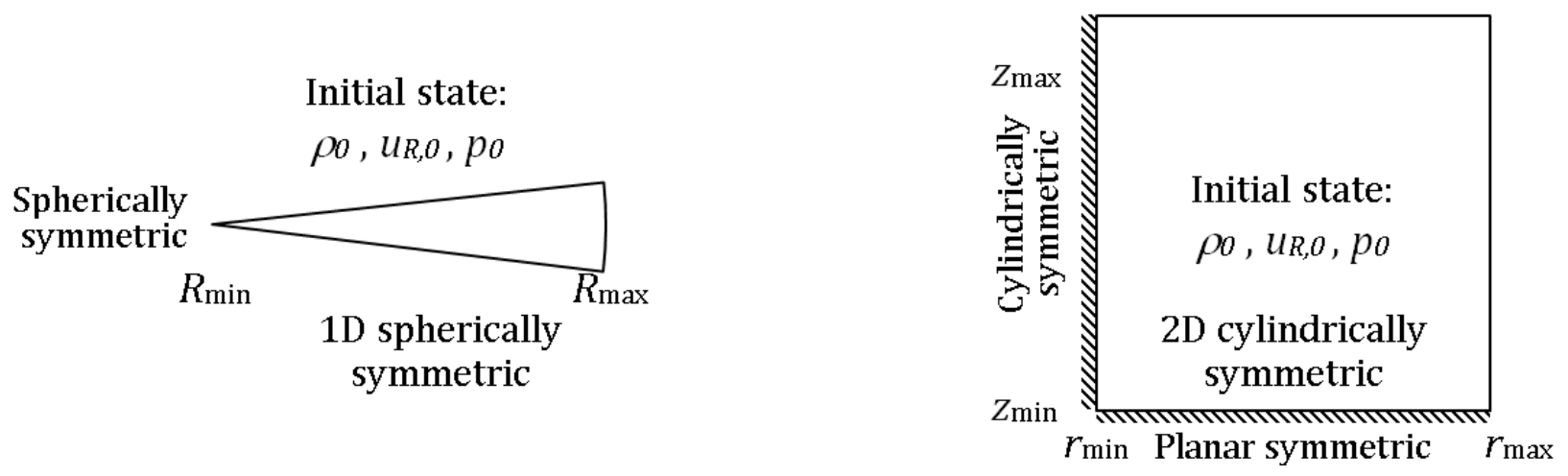

Fig. 6.1: Initial configuration of Guderley Problem.

\subsection{Problem Parameters}

Table 6.1: Parameters for the Guderley problem.

\begin{tabular}{|l|l|l|l|l|l|l|l|}
\hline$t_{\text {init }}$ & $t_{\text {fin }}$ & $\gamma$ & $R_{0}$ & $\rho_{0}$ & $u_{0}$ & $p_{0}$ & $e_{0}$ \\
\hline$[\mathrm{s}]$ & {$[\mathrm{s}]$} & {$[-]$} & {$[\mathrm{cm}]$} & {$\left[\mathrm{g} / \mathrm{cm}^{3}\right]$} & {$[\mathrm{cm} / \mathrm{s}]$} & {$\left[\mathrm{dyn} / \mathrm{cm}^{2}\right]$} & {$[\mathrm{erg} / \mathrm{g}]$} \\
\hline-1 & 0.5 & 3 & 1.0 & 1.0 & 0.0 & $2 \times 10^{-12}$ & $1 \times 10^{-12}$ \\
\hline
\end{tabular}

\subsection{Initial Conditions}

Uniform, constant material density and velocity in the spherical region $0 \leq R<R_{0}$ according to the Parameters table. States are related through the polytropic (ideal gas) EOS: $p=(\gamma-1) \rho e$, where, $\gamma=3$ is the (constant) ratio of specific heats. For $R>R_{0}$, the initial state is evaluated numerically and must be assigned to the mesh. 


\subsection{Boundary Conditions}

The problem is to be run so that any spurious waves generated by boundary conditions do not affect the solution on the Comparison Domain, defined below. Possible boundary conditions are:

Inner: $R_{\min }, r_{\min }, z_{\min }:$ Symmetric

Outer: $R_{\max }, r_{\max }, z_{\max }:$ Constant in time

\subsection{Mesh}

Region Boundaries ${ }^{1}$ :

1D: $R_{\min }=0.0, R_{\max }=3.0 \mathrm{~cm}$

2D: $(r, z)_{\min }=0.0,(r, z)_{\max }=3.0 \mathrm{~cm}$

Mesh Resolutions:

1D spherical: $N_{R}=60,120,240,480,960 ; \Delta_{R}=0.05,0.025,0.0125,0.00625,0.003125$

2D cylindrical: $N_{r}=N_{z}=60,120,240,480,960 ; \Delta_{r}=\Delta_{z}=0.05,0.025,0.0125,0.00625,0.003125$

\subsection{Output}

In ASCII comma- or space-delimited format, ${ }^{2}$ to include:

1. Values of density, velocity, pressure, SIE as a function of position at $t_{0}$ and $t_{\text {fin }}$.

2. Entire mesh total energy, kinetic energy, internal energy as a function of time.

\subsection{Comparison Domain}

1D: $R \in[0,1]$

2D: $(r, z) \in[0,1] \times[0,1]$

\footnotetext{
${ }^{1}$ Here, $R=$ spherical radial coordinate, with $R=r^{2}+z^{2}$, where $r=$ cylindrical radial coordinate.

${ }^{2}$ Sample output available upon request.
} 
6.8 Results at $t_{\text {fin }}=0.6 \mathbf{s}$
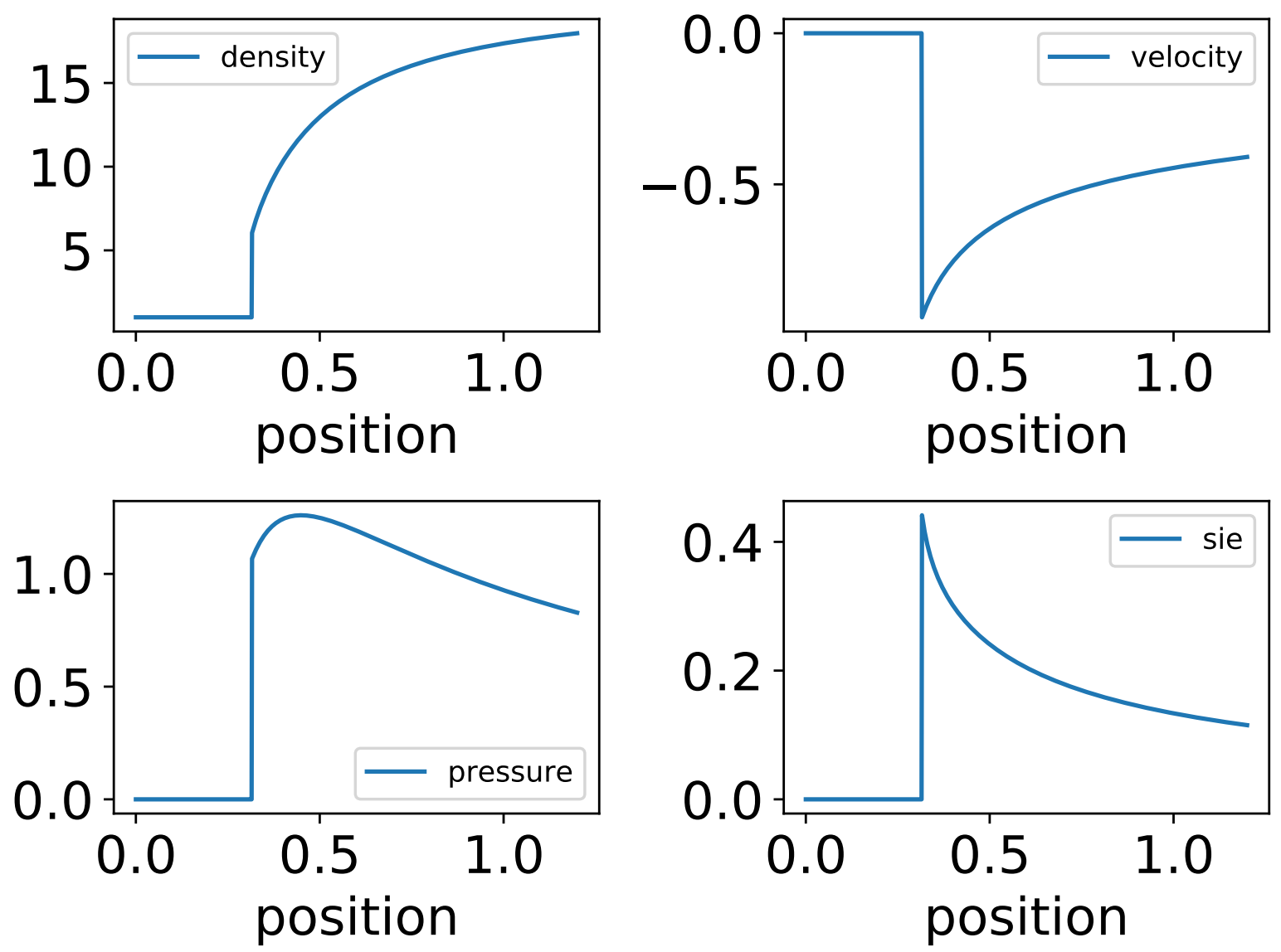


\subsection{Description}

1D JWL Riemann Problems are finite-dimensional restrictions of mathematically ideal, infinite domain shock tube experiments with materials described by the JWL EOS. At $t=0$, the states are constant and uniform and are separated by a massless interface. The removal of the interface leads to the evolution of the self-similar solution, consisting of some combination of shock, contact, and rarefaction waves.

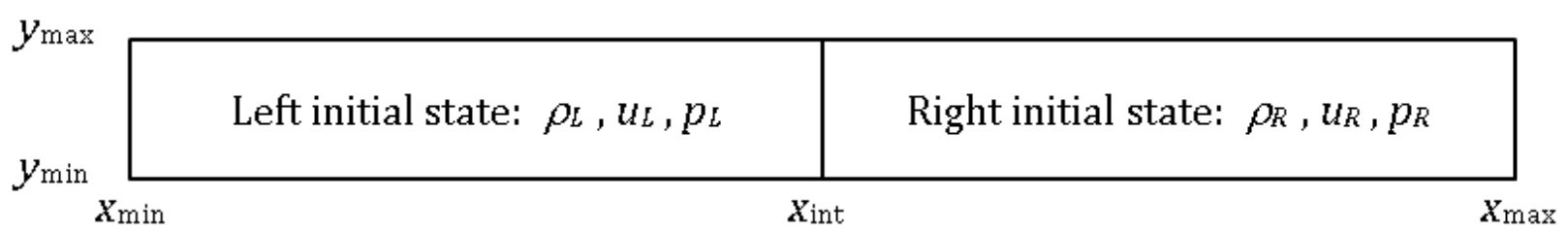

Fig. 7.1: Initial geometetry of the 1D JWL Riemann problems.

\subsection{Problem Parameters}

The tests numbers in the parameter tables below correspond to the following tests [Kamm],

1. Shyue shock tube: A shock tube problem with the cannonical rarefaction-contact-shock structure witha a JWL EOS for TNT [Shyue].

2. Lee shock tube:: A shock tube problem with a shock-contact-rarefaction structure with a JWL EOS for LX-17 [Lee].

Table 7.1: Global parameters for the JWL Riemann problems.

\begin{tabular}{|l|l|l|l|}
\hline Test & $x_{\text {int }}$ & $t_{\text {fin }}$ & JWL \\
\hline & {$[\mathrm{cm}]$} & {$[\mu \mathrm{s}]$} & $\mathrm{EOS}$ \\
\hline 1 & 50.0 & 12.0 & $\# 1$ \\
\hline 2 & 50.0 & 20.0 & $\# 2$ \\
\hline
\end{tabular}


Table 7.2: Left and Right parameters for the JWL Riemann problems.

\begin{tabular}{|l|l|l|l|l|l|}
\hline$\rho_{L}$ & $u_{L}$ & $p_{L}$ & $\rho_{R}$ & $u_{R}$ & $p_{R}$ \\
\hline$\left[\mathrm{g} / \mathrm{cm}^{3}\right]$ & {$[\mathrm{cm} / \mu \mathrm{s}]$} & {$[\mathrm{Mbar}]$} & {$\left[\mathrm{g} / \mathrm{cm}^{3}\right]$} & {$[\mathrm{cm} / \mu \mathrm{s}]$} & {$[\mathrm{Mbar}]$} \\
\hline 1.7 & 0.0 & 10.0 & 1.0 & 0.0 & 0.5 \\
\hline 0.9525 & 0.0 & 1.0 & 3.810 & 0.0 & 2.0 \\
\hline
\end{tabular}

\subsection{Initial conditions}

Uniform and constant material density, pressure, and velocity on each side of the initial interface ( $\mathrm{x}=\mathrm{xint})$. Other states are related through the JWL equation of state, which can be expressed in the general Mie-Grüneissen form shown below, with the JWL EOS paramters given in the table below ${ }^{1}$.

$$
p=p_{\text {ref }}(\rho)+\Gamma(\rho) \rho\left[e-e_{\text {ref }}(\rho)\right]
$$

with

$$
\begin{gathered}
\Gamma(\rho)=\Gamma_{0} \\
p_{r e f}(\rho)=A \exp \left(R_{1} \rho_{0} / \rho\right)+B \exp \left(-R_{2} \rho_{0} / \rho\right)
\end{gathered}
$$

and,

$$
e_{r e f}(\rho)=\left[A /\left(R_{1} \rho_{0}\right)\right] \exp \left(-R_{1} \rho_{0} / \rho\right)+\left[B /\left(R_{2} \rho_{0}\right)\right] \exp \left(-R_{2} \rho_{0} / \rho\right)-e_{0}
$$

Table 7.3: JWL EOS parameters for the 1D JWL Riemann problems.

\begin{tabular}{|l|l|l|l|l|l|l|l|l|}
\hline JWL & Material & $\rho_{0}$ & $e_{0}$ & $\Gamma_{0}$ & $A$ & $B$ & $R_{1}$ & $R_{2}$ \\
\hline EOS & & {$\left[\mathrm{g} / \mathrm{cm}^{3}\right]$} & {$\left[\right.$ Mbar-cm $\left.^{3} / \mathrm{g}\right]$} & {$[-]$} & {$[$ Mbar $]$} & {$[$ Mbar $]$} & {$[-]$} & {$[-]$} \\
\hline$\# 1$ & TNT & 1.84 & 1.7 & 0.25 & 8.545 & 0.205 & 4.6 & 1.35 \\
\hline$\# 2$ & LX-17 & 1.905 & 0.9525 & 0.8938 & 632.1 & -0.04472 & 11.3 & 1.13 \\
\hline
\end{tabular}

\subsection{Boundary conditions}

Left/right: $x_{\min }, x_{\max }$ : Constant in time

Top/Bottom: $y_{\min }, y_{\max }$ : Reflective

\subsection{Mesh}

For all problems, $x_{\min }=0.0$ and $x_{\max }=100.0 \mathrm{~cm}$; in $2 \mathrm{D}, y_{\min }=0.0, y_{\max }=20.0 \mathrm{~cm}$.

1D: $N_{x}=50,100,200,400,800$

2D: $\left(N_{x}, N_{y}\right)=(50,10),(100,20),(200,40),(400,80),(800,160)$

\footnotetext{
${ }^{1}$ The pressures developed in these tests may not be consistent with realistic problems.
} 


\subsection{Output}

In ASCII comma- or space-delimited format ${ }^{2}$, to include:

1. Values of density, velocity, pressure, SIE as a function of position at $t_{0}$ and $t_{\text {fin }}$.

2. Entire mesh total energy, kinetic energy, internal energy as a function of time.

\subsection{Comparison Domain}

1D: $x \in[0,100]$

2D: $(x, y) \in[0,100] \times[0,20]$

\subsection{Exact Solutions at $t_{\mathrm{fin}, 0}=12$ and $t_{\mathrm{fin}, 1}=20 \mu \mathrm{s}$}

The exact solutions at $t_{\text {fin }}$ for both JWL Riemann problems are plotted below:



${ }^{2}$ Sample output available upon request. 


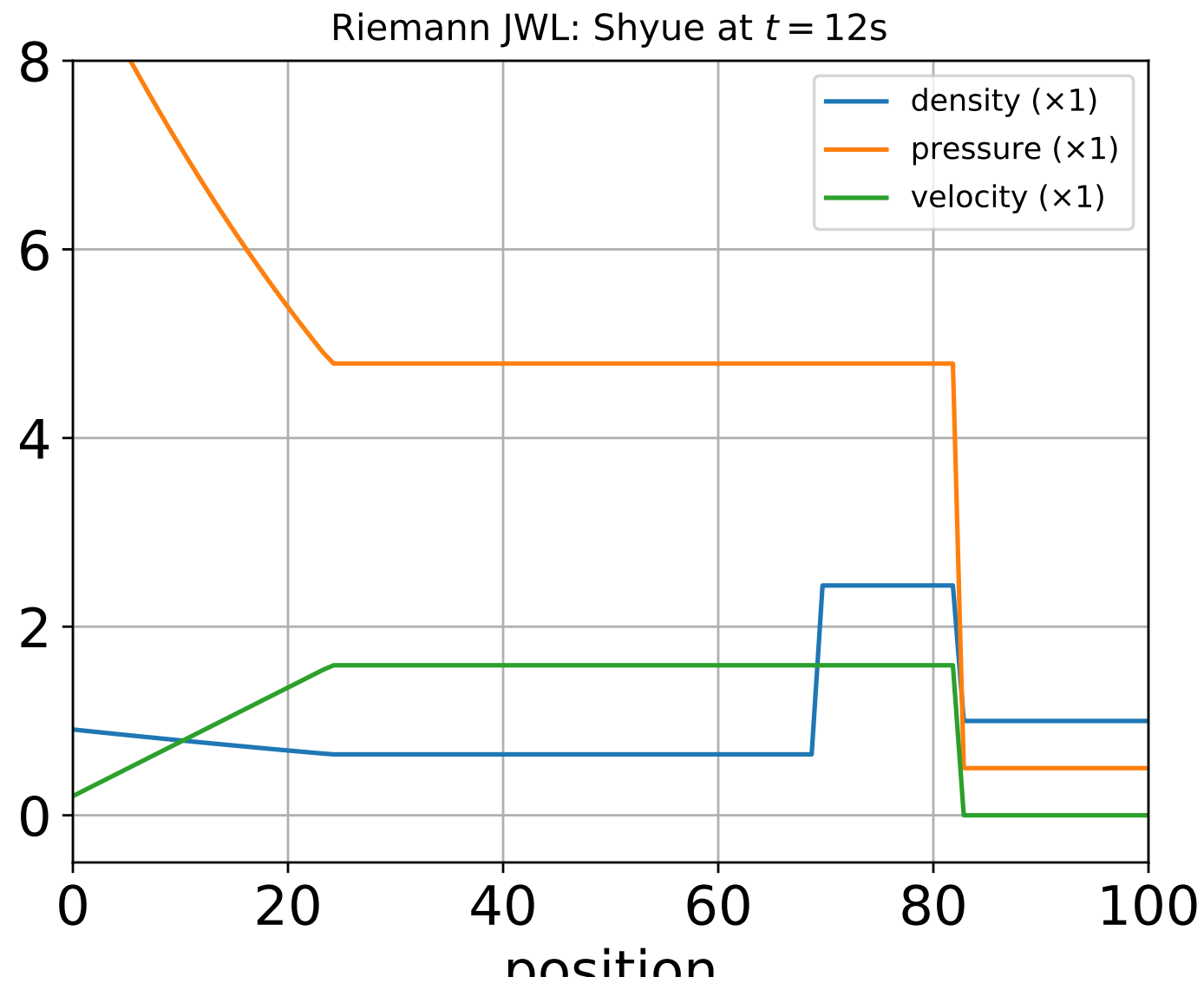




\subsection{Description}

The Kenamond High Explosive Problem Set is a series of problems designed to test the burn table solution (HE light times) generated for programmed burn simulations. The suite of test problems has exact solutions in 2D and 3D [Kenamond].

It should be understood that these burn time calculations are purely geometry-based solutions. They do not account for HE behaviors such as shock formation time, inert boundary behaviors or behavior at boundaries between two HEs.

The Kenamond HE Problem 1 is used to test a code's ability to calculate burn tables for an unobstructed line-of-sight, single-point initiation of a single HE region. The general problem is described as follows:

An infinite medium of a single HE with constant detonation velocity, $D$, is ignited at time $t=t_{d}$ by a single point detonator located at $\vec{x}=\vec{x}_{d}$. The calculated burn-time solution should depend only on these three values. The specific values used in the verification calculation are specified in the Parameters table in the Problem Parameters section.

A Cartesian mesh is used for the verification calculation. Details are provided in the Mesh section. The mesh is not equally spaced in all directions, which highlights the truncation errors in a given solution method.



Fig. 8.1: Initial configuration of 2D and 3D Kenamond HE Problem 1.

\subsection{Solution}

The HE light time solution for spherical propagation at the specified detonation velocity, ignition time and detonation location is independent of the inital state parameters specified for the HE material and depends only on these three quantities. The solution for the specific problem considered is

$$
t(\vec{x})=\|\vec{x}\|
$$




\subsection{Problem Parameters}

Table 8.1: Parameters for the Kenamond HE Problem 1

\begin{tabular}{|l|l|l|l|}
\hline$D$ & $t_{d}$ & $\vec{x}_{d}$ & $u_{0}$ \\
\hline$[\mathrm{cm} / \mu \mathrm{s}]$ & {$[\mu \mathrm{s}]$} & {$[\mathrm{cm}]$} & {$[\mathrm{cm} / \mu \mathrm{s}]$} \\
\hline 1.0 & 0 & 2D:(0.0,0.0) & 0.0 \\
\hline & & 3D:(0.0,0.0,0.0) & \\
\hline
\end{tabular}

\subsection{Initial Conditions}

Uniform and constant HE detonation velocity ${ }^{1}$. Specified detonation location and ignition time. No initial material velocity. Uniform and constant material density and pressure. JWL EOS for HE materials.

For verification purposes, the specific HE used is PBX-9501². JWL EOS parameters for PBX-9501 are shown in the HE parameters table below [Dobratz].

Table 8.2: HE parameters for the Kenamond HE Problem 1

\begin{tabular}{|l|l|l|l|l|l|l|l|l|l|l|}
\hline$\rho_{0}$ & $P$ & $D$ & $E_{0}$ & $\Gamma$ & $A$ & $B$ & $C$ & $R_{1}$ & $R_{2}$ & $\omega$ \\
\hline$\left[\mathrm{g} / \mathrm{cm}^{3}\right]$ & {$[\mathrm{Mbar}]$} & {$[\mathrm{cm} / \mu \mathrm{s}]$} & {$[\mathrm{Mbar}]$} & {$[-]$} & {$[\mathrm{Mbar}]$} & {$[\mathrm{Mbar}]$} & {$[\mathrm{Mbar}]$} & {$[-]$} & {$[-]$} & {$[-]$} \\
\hline 1.840 & 0.370 & 0.88 & 0.1020 & 2.851 & 8.524 & 0.1802 & 0.01207 & 4.55 & 1.30 & 0.38 \\
\hline
\end{tabular}

\subsection{Boundary Conditions}

The problem is to be run so that any spurious waves generated by boundary conditions do not affect the solution on the Comparison Domain, defined below ${ }^{3}$. Possible boundary conditions are:

Inner: $x_{\min }, y_{\min }, z_{\min }:$ Symmetric

Outer: $x_{\max }, y_{\max }, z_{\max }:$ Free

\subsection{Mesh}

Region Boundaries:

2D: $x_{\min }=y_{\min }=0.0, x_{\max }=y_{\max }=10.0 \mathrm{~cm}$

3D: $x_{\min }=y_{\min }=z_{\min }=0.0, x_{\max }=y_{\max }=z_{\max }=10.0 \mathrm{~cm}$

Mesh Resolutions:

2D Cartesian:

$$
\begin{aligned}
& N_{x}=20,40,80,160,320 ; \Delta x=0.5,0.25,0.125,0.0625,0.03125 \mathrm{~cm} \\
& N_{y}=40,80,160,320,640 ; \Delta y=0.25,0.125,0.0625,0.03125,0.015625 \mathrm{~cm}
\end{aligned}
$$

\footnotetext{
${ }^{1}$ The detonation velocity used for the prescribed problem is not thermodynamically consistent with the detonation velocity of PBX-9501. The prescribed detonation velocity was chosen to simplify the solution.

${ }^{2} \mathrm{HE}$ light times should not be dependent on HE material parameters, only the detonation velocity.

${ }^{3}$ Boundary conditions should not affect the burn time solution.
} 
3D Cartesian:

$$
\begin{aligned}
& N_{x}=N_{z}=20,40,80,160 ; \Delta x=\Delta z=0.5,0.25,0.125,0.0625 \mathrm{~cm} \\
& N_{y}=40,80,160,320 ; \Delta y=0.25,0.125,0.0625,0.03125 \mathrm{~cm}
\end{aligned}
$$

\subsection{Output}

In ASCII comma- or space-delimited format ${ }^{4}$, to include:

1. Burn time as a function of position.

\subsection{Comparison Domain}

2D: $(x, y) \in[0.0,10.0] \times[0.0,10.0]$

3D: $(x, y, z) \in[0.0,10.0] \times[0.0,10.0] \times[0.0,10.0]$

No simulation time steps are necessary for this calculation, since the burn times are calculated at the beginning of a run. One time step may be necessary in a particular code to obtain the desired output.

\footnotetext{
${ }^{4}$ Sample output available upon request.
} 


\subsection{Exact Solution}
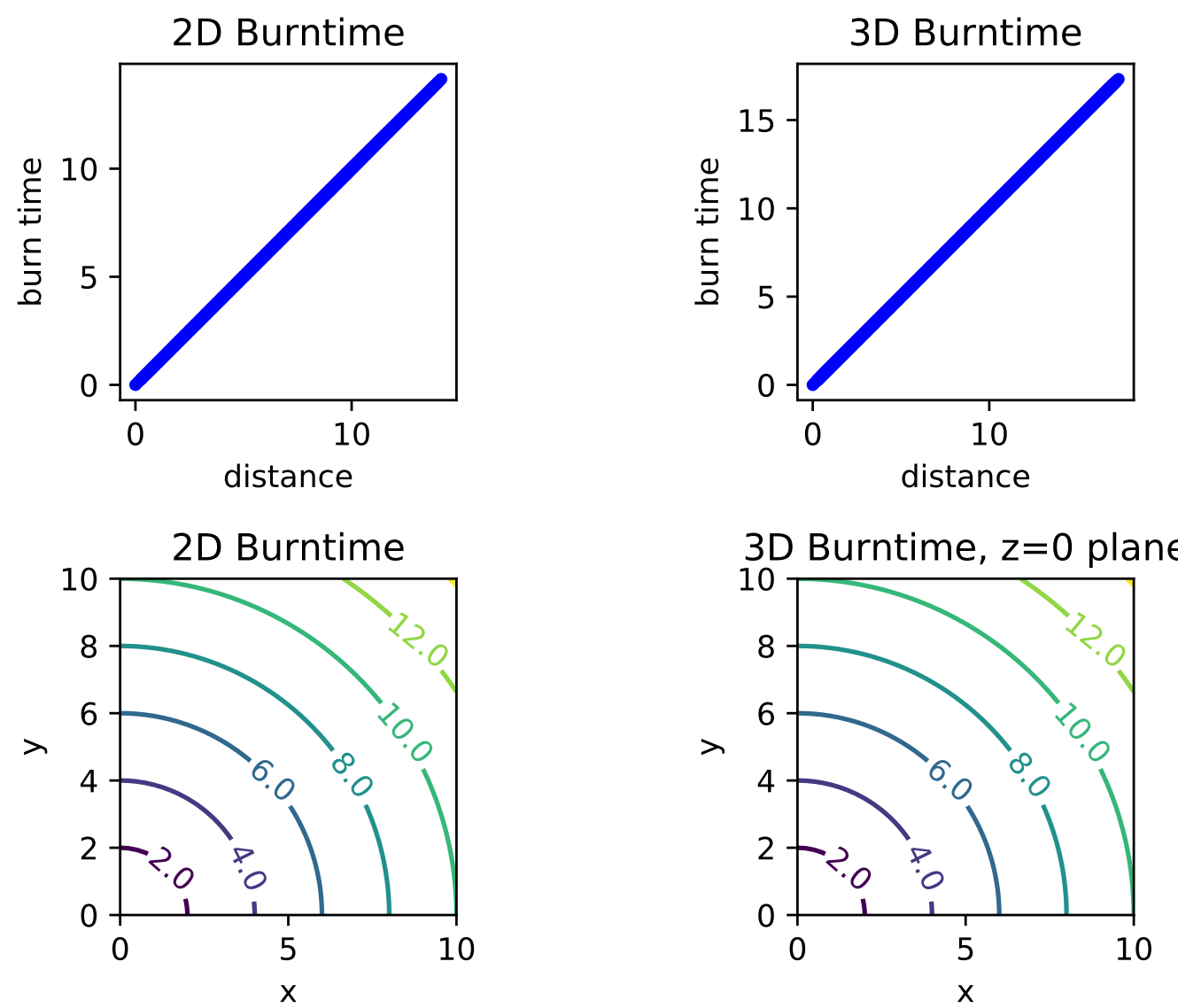


\subsection{Description}

The Kenamond High Explosive Problem Set is a series of problems designed to test the burn table solution (HE light times) generated for programmed burn simulations. The suite of test problems has exact solutions in 2D and 3D [Kenamond].

It should be understood that these burn time calculations are purely geometry-based solutions. They do not account for HE behaviors such as shock formation time, inert boundary behaviors or behavior at boundaries between two HEs.

The Kenamond HE Problem 2 is used to test a code's ability to calculate burn tables for an unobstructed line-of-sight, multi-point initiation of a region composed of two HE materials with different detonation velocities. The problem is described as follows:

An HE sphere of radius $R$ centered at the origin with constant detonation velocity $D_{1}$ is surrounded by an infinite medium of a second $\mathrm{HE}$ with constant detonation velocity $D_{2}$. The specific values used for these three quantities in the verification calculation are specified in the Parameters table in the Problem Parameters section. Five point detonators located at $\vec{x}=\vec{x}_{d_{i}}$ are ignited at times $t=t_{d_{i}}$. The detonators, which are located on the $y$-axis in the 2D test and on the $z$-axis in the 3D test, are specified in the Detonator specifications table in the Problem Parameters section.

An $(r, \theta)$ mesh is used for the 2D verification calculation. An $(r, \theta, \phi)$ mesh is used for the 3D test. Details are provided in the Mesh section.

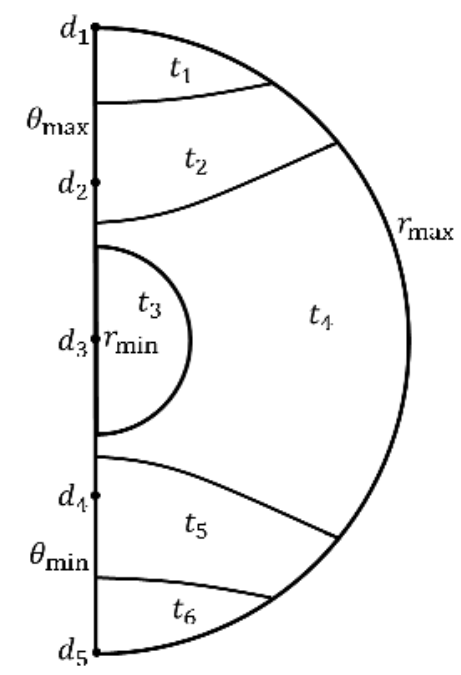

Fig. 9.1: Initial configuration of 2D Kenamond HE Problem 2. The 3D problem is revolved to form a quarter of a sphere. 


\subsection{Solution}

The HE light time solution is independent of the initial state parameters specified for the HE material and depends only on the specified detonation velocities, ignition times and detonator locations.

2D Specific Solution: For the specific $2 D$ case considered, the solution at the point $\vec{p}=(x, y)$ is as follows:

$$
t(\vec{p})=\min \left(t_{1}, t_{2}, \max \left(t_{3}, t_{4}\right), t_{5}, t_{6}\right)
$$

where,

$$
\begin{array}{r}
t_{1}(\vec{p})=\sqrt{x^{2}+(y-10)^{2}}+2 \\
t_{2}(\vec{p})=\sqrt{x^{2}+(y-5)^{2}}+1 \\
t_{3}(\vec{p})=\frac{\sqrt{x^{2}+y^{2}}}{2} \\
t_{4}(\vec{p})=\sqrt{x^{2}+y^{2}}-1.5 \\
t_{5}(\vec{p})=\sqrt{x^{2}+(y+5)^{2}}+1 \\
t_{6}(\vec{p})=\sqrt{x^{2}+(y+10)^{2}}+2
\end{array}
$$

3D Specific Solution: For the specific $2 D$ case considered, the solution at the point $\vec{p}=(x, y, z)$ is as follows:

$$
t(\vec{p})=\min \left(t_{1}, t_{2}, \max \left(t_{3}, t_{4}\right), t_{5}, t_{6}\right)
$$

where,

$$
\begin{array}{r}
t_{1}(\vec{p})=\sqrt{x^{2}+y^{2}+(z-10)^{2}}+2 \\
t_{2}(\vec{p})=\sqrt{x^{2}+y^{2}+(z-5)^{2}}+1 \\
t_{3}(\vec{p})=\frac{\sqrt{x^{2}+y^{2}+z^{2}}}{2} \\
t_{4}(\vec{p})=\sqrt{x^{2}+y^{2}+z^{2}}-1.5 \\
t_{5}(\vec{p})=\sqrt{x^{2}+y^{2}+(z+5)^{2}}+1 \\
t_{6}(\vec{p})=\sqrt{x^{2}+y^{2}+(z+10)^{2}}+2
\end{array}
$$

The regions in the figure above are labeled by the time solution valid in that region.

\subsection{Problem Parameters}

Table 9.1: Parameters for the Kenamond HE Problem 2

\begin{tabular}{|l|l|l|l|}
\hline$R$ & $D_{1}$ & $D_{2}$ & $u_{0}$ \\
\hline$[\mathrm{cm}]$ & {$[\mathrm{cm} / \mu \mathrm{s}]$} & {$[\mathrm{cm} / \mu \mathrm{s}]$} & {$[\mathrm{cm} / \mu \mathrm{s}]$} \\
\hline 3.0 & 2.0 & 1.0 & 0.0 \\
\hline
\end{tabular}


Table 9.2: Detonator specifications (in Cartesian coordinates) for the Kenamond HE Problem 2

\begin{tabular}{|l|l|l|}
\hline Detonator & $t_{d_{i}}$ & $\vec{x}_{d_{i}}$ \\
\hline$\left(d_{i}\right)$ & {$[\mu \mathrm{s}]$} & {$[\mathrm{cm}]$} \\
\hline 1 & 2.0 & 2D: $(0.0,10.0)$ \\
\hline & & 3D: $(0.0,0.0,10.0)$ \\
\hline 2 & 1.0 & 2D: $(0.0,5.0)$ \\
\hline & & 3D: $(0.0,0.0,5.0)$ \\
\hline 3 & 0.0 & 2D: $(0.0,0.0)$ \\
\hline & & 3D: $(0.0,0.0,0.0)$ \\
\hline 4 & 1.0 & 2D: $(0.0,-5.0)$ \\
\hline & & 3D: $(0.0,0.0,-5.0)$ \\
\hline 5 & 2.0 & 2D: $(0.0,-10.0)$ \\
\hline & & 3D: $(0.0,0.0,-10.0)$ \\
\hline
\end{tabular}

\subsection{Initial Conditions}

Uniform and constant HE detonation velocity ${ }^{1}$. Specified detonation locations and ignition times. No initial material velocity. Uniform and constant material density and pressure. JWL EOS for HE materials.

For verification purposes, the specific HEs used are PBX-9501 (HE1) and Comp B (HE2)2. JWL EOS parameters for PBX-9501 and Comp B are shown in the HE parameters below [Dobratz].

Table 9.3: HE parameters for the Kenamond HE Problem 2.

\begin{tabular}{|l|l|l|l|l|l|l|l|l|l|l|}
\hline$\rho_{0}$ & $P$ & $D$ & $E_{0}$ & $\Gamma$ & $A$ & $B$ & $C$ & $R_{1}$ & $R_{2}$ & $\omega$ \\
\hline$\left[\mathrm{g} / \mathrm{cm}^{3}\right]$ & {$[\mathrm{Mbar}]$} & {$[\mathrm{cm} / \mu \mathrm{s}]$} & {$[\mathrm{Mbar}]$} & {$[-]$} & {$[\mathrm{Mbar}]$} & {$[\mathrm{Mbar}]$} & {$[\mathrm{Mbar}]$} & {$[-]$} & {$[-]$} & {$[-]$} \\
\hline 1.840 & 0.370 & 0.88 & 0.1020 & 2.851 & 8.524 & 0.1802 & 0.01207 & 4.55 & 1.30 & 0.38 \\
\hline 1.717 & 0.295 & 0.798 & 0.0850 & 2.706 & 5.242 & 0.07678 & 0.01082 & 4.20 & 1.10 & 0.34 \\
\hline
\end{tabular}

\subsection{Boundary Conditions}

The problem is to be run so that any spurious waves generated by boundary conditions do not affect the solution on the Comparison Domain, defined below ${ }^{3}$. Possible boundary conditions are:

Inner: $r_{\min }:$ Fixed, $\theta_{\min }:$ Symmetric, $\phi_{\min }:$ Fixed

Outer: $r_{\max }:$ Free, $\theta_{\max }:$ Symmetric, $\phi_{\max }:$ Fixed

\subsection{Mesh}

\section{Region Boundaries ${ }^{4}$ :}

\footnotetext{
${ }^{1}$ The detonation velocities used for the prescribed problem are not thermodynamically consistent with the detonation velocities of PBX-9501 and Comp B. The prescribed detonation velocities were chosen to simplify the solution.

${ }^{2}$ He light times should not be dependent on HE material parameters, only the detonation velocity.

${ }^{3}$ Boundary conditions should not affect the burn time solution.

${ }^{4} r$ represents the polar radius in 2D and the spherical radius in 3D. $\theta$ represents the azimuthal angle in both $2 \mathrm{D}$ and $3 \mathrm{D}$. $\phi$ represents the polar angle in $3 \mathrm{D}$.
} 
2D: $r_{\min }=0.0 \mathrm{~cm}, \theta_{\min }=-\pi / 2$ radians; $r_{\max }=10.0 \mathrm{~cm}, \theta_{\max }=\pi / 2$ radians

3D: $r_{\min }=0.0 \mathrm{~cm}, \theta_{\min }=0.0$ radians, $\phi_{\min }=0.0$ radians; $r_{\max }=10.0 \mathrm{~cm}, \theta_{\max }=\pi / 2$ radians, $\phi_{\max }=\pi$ radians Mesh Resolutions:

2D Polar:

$$
\begin{aligned}
& N_{r}=40,80,160,320 ; \Delta r=0.25,0.125,0.0625,0.03125 \mathrm{~cm} \\
& N_{\theta}=64,128,256,512 ; \Delta \theta=\pi / 64, \pi / 128, \pi / 256, \pi / 512 \text { radians }
\end{aligned}
$$

3D Spherical:

$$
\begin{aligned}
& N_{r}=40,80,160 ; \Delta r=0.25,0.125,0.0625 \mathrm{~cm} \\
& N_{\theta}=8,16,32 ; \Delta \theta=\pi / 16, \pi / 32, \pi / 64 \text { radians } \\
& N_{\phi}=64,128,256 ; \Delta \phi=\pi / 64, \pi / 128, \pi / 256 \text { radians }
\end{aligned}
$$

\subsection{Output}

In ASCII comma- or space-delimited format ${ }^{5}$, to include:

1. Burn time as a function of position.

\subsection{Comparison Domain}

2D: $(r, \theta) \in[0.0,10.0] \times[-\pi / 2, \pi / 2]$

3D: $(r, \theta, \phi) \in[0.0,10.0] \times[0.0, \pi / 2] \times[0.0, \pi]$

No simulation time steps are necessary for this calculation, since the burn times are calculated at the beginning of a run. One time step may be necessary in a particular code to obtain the desired output.

\footnotetext{
${ }^{5}$ Sample output available upon request.
} 


\subsection{Exact Solution}

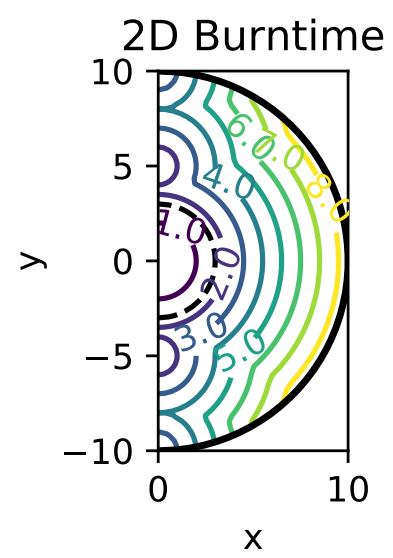

3D Burntime, theta $=\mathrm{pi} / 2$ plane

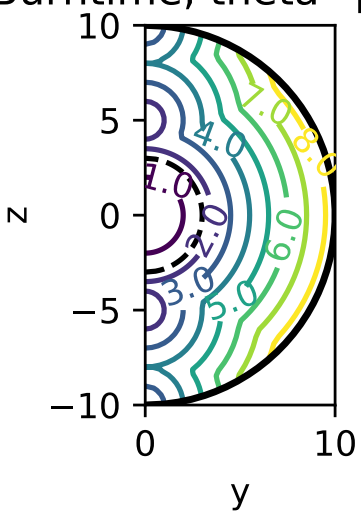

3D Burntime, theta $=0$ plane
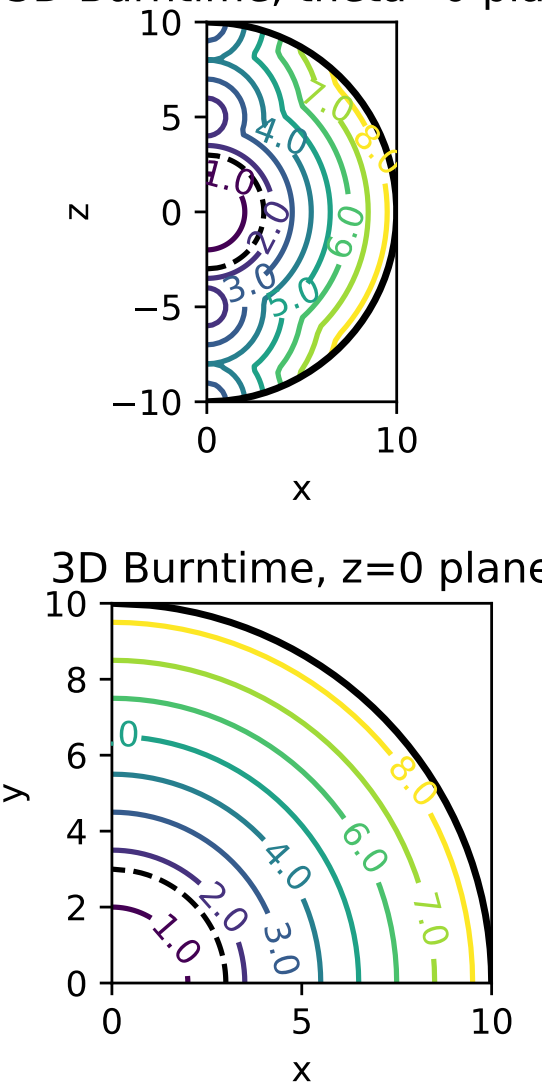


\subsection{Description}

The Kenamond High Explosive Problem Set is a series of problems designed to test the burn table solution of HE light times generated for programmed burn simulations. The suite of test problems has exact solutions in 2D and 3D [Kenamond].

It should be understood that these burn time calculations are purely geometry-based solutions. They do not account for HE behaviors such as shock formation time, inert boundary behaviors or behavior at boundaries between two HEs.

The Kenamond HE Problem 3 is used to test a code's ability to calculate burn tables for a single-point initiation of a single HE region surrounding an inert region. The problem is described as follows:

An infinite medium of a single HE with constant detonation velocity, $D$, surrounds an inert obstacle of radius, $R$, centered at the origin. A single point detonator located at $\vec{x}_{d}$ is ignited at time $t=t_{d}$. The detonator is located on the $y$-axis in the $2 \mathrm{D}$ test and on the $z$-axis in the 3D test. The calculated burn-time solution should depend only on these four values. The specific values used in the verification calculation are specified in the Parameters table in the Problem Parameters section.

An $(r, \theta)$ mesh is used for the 2D test. An $(r, \theta, \phi)$ mesh is used for the 3D test. Details are provided in the Mesh section.

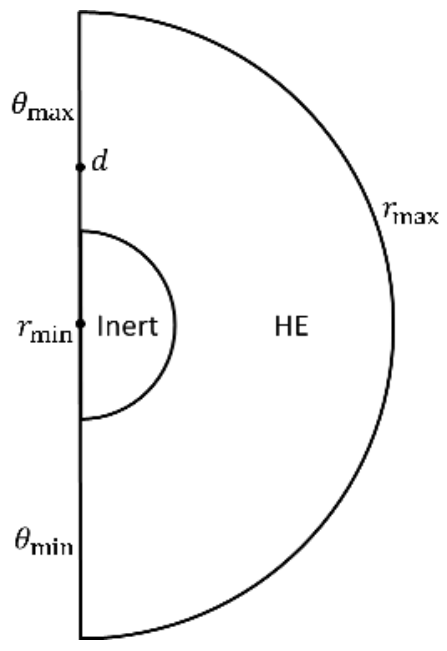

Fig. 10.1: Initial configuration of 2D Kenamond HE Problem 3. The 3D problem is revolved to form a quarter of a sphere. 


\subsection{Solution}

The HE light time solution is independent of the inital state parameters specified for the HE material and depends only on the size of the inert object, the specified detonation time and position, and the HE detonation velocity. The HE material can be divided into two solution regions: the material in the line-of-sight of the detonator and the material in the shadow of the inert object. See the second figure below, where $t_{1}$ designates the line-of-sight region and $t_{2}$ designates the shadow region.

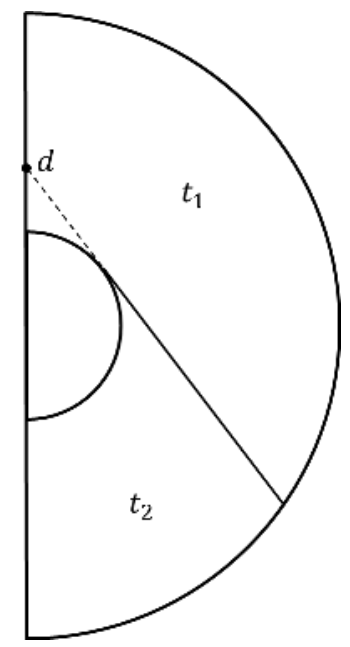

Fig. 10.2: The two solution regions for the Kenamond HE Problem 3. $t_{1}$ designates the line-of-sight region and $t_{2}$ designates the shadow region.

2D Specific Solution: For the specific $2 D$ case considered, the solution at the point $\vec{p}=(x, y)$ is as follows:

$$
t(\vec{p})= \begin{cases}t_{1} & \text { if } \theta \leq 0 \\ t_{2} & \text { if } \theta>0\end{cases}
$$

where,

$$
\begin{array}{r}
t_{1}(\vec{p})=\frac{\sqrt{x^{2}+(y-5)^{2}}}{2} \\
t_{2}(\vec{p})=2+\frac{3}{2} \theta+\frac{\sqrt{x^{2}+y^{2}-9}}{2} \\
\theta=\pi-\arccos \left(-\frac{y}{\sqrt{x^{2}+y^{2}}}\right)-\arccos \left(\frac{3}{\sqrt{x^{2}+y^{2}}}\right)-\arccos \left(\frac{3}{5}\right)
\end{array}
$$

3D Specific Solution: In the 3D problem, the 2D $y$-axis becomes the 3D $z$-axis, while the 2D $x$-axis becomes the cylindrical $r$-axis $\left(r^{2}=x^{2}+y^{2}\right)$. For the specific $3 D$ case considered, the solution at the point $\vec{p}=(x, y, z)$ is as follows:

$$
t(\vec{p})= \begin{cases}t_{1} & \text { if } \theta \leq 0 \\ t_{2} & \text { if } \theta>0\end{cases}
$$


where,

$$
\begin{array}{r}
t_{1}(\vec{p})=\frac{\sqrt{x^{2}+y^{2}+(z-5)^{2}}}{2} \\
t_{2}(\vec{p})=2+\frac{3}{2} \theta+\frac{\sqrt{x^{2}+y^{2}+z^{2}-9}}{2} \\
\theta=\pi-\arccos \left(-\frac{z}{\sqrt{x^{2}+y^{2}+z^{2}}}\right)-\arccos \left(\frac{3}{\sqrt{x^{2}+y^{2}+z^{2}}}\right)-\arccos \left(\frac{3}{5}\right)
\end{array}
$$

\subsection{Problem Parameters}

Table 10.1: Parameters for the Kenamond HE Problem 3

(Detonator positions are given in Cartesian coordinates)

\begin{tabular}{|l|l|l|l|l|}
\hline$R$ & $D$ & $t_{d}$ & $\vec{x}_{d}$ & $u_{0}$ \\
\hline$[\mathrm{cm}]$ & {$[\mathrm{cm} / \mu \mathrm{s}]$} & {$[\mu \mathrm{s}]$} & {$[\mathrm{cm}]$} & {$[\mathrm{cm} / \mu \mathrm{s}]$} \\
\hline 3.0 & 2.0 & 0.0 & 2D: $(0.0,5.0)$ & 0.0 \\
\hline & & & 3D: $(0.0,0.0,5.0)$ & \\
\hline
\end{tabular}

\subsection{Initial Conditions}

Uniform and constant HE detonation velocity ${ }^{1}$. Specified detonation location and ignition time. No initial material velocity. Uniform and constant material density and pressure. JWL EOS for HE materials.

For verification purposes, the specific HE used is PBX-9501². JWL EOS parameters for PBX-9501 are shown in the HE parameters table below [Dobratz].

Table 10.2: HE parameters for the Kenamond HE Problem 3

\begin{tabular}{|l|l|l|l|l|l|l|l|l|l|l|}
\hline$\rho_{0}$ & $P$ & $D$ & $E_{0}$ & $\Gamma$ & $A$ & $B$ & $C$ & $R_{1}$ & $R_{2}$ & $\omega$ \\
\hline$\left[\mathrm{g} / \mathrm{cm}^{3}\right]$ & {$[\mathrm{Mbar}]$} & {$[\mathrm{cm} / \mu \mathrm{s}]$} & {$[\mathrm{Mbar}]$} & {$[-]$} & {$[\mathrm{Mbar}]$} & {$[\mathrm{Mbar}]$} & {$[\mathrm{Mbar}]$} & {$[-]$} & {$[-]$} & {$[-]$} \\
\hline 1.840 & 0.370 & 0.88 & 0.1020 & 2.851 & 8.524 & 0.1802 & 0.01207 & 4.55 & 1.30 & 0.38 \\
\hline
\end{tabular}

\subsection{Boundary Conditions}

The problem is to be run so that any spurious waves generated by boundary conditions do not affect the solution on the Comparison Domain, defined below ${ }^{3}$. Possible boundary conditions are:

Inner: $r_{\min }:$ Fixed, $\theta_{\min }:$ Symmetric, $\phi_{\min }:$ Fixed

Outer: $r_{\max }:$ Free, $\theta_{\max }:$ Symmetric, $\phi_{\max }:$ Fixed

\footnotetext{
${ }^{1}$ The detonation velocity used for the prescribed problem is not thermodynamically consistent with the detonation velocity of PBX-9501. The prescribed detonation velocity was chosen to simplify the solution.

${ }^{2}$ He light times should not be dependent on HE material parameters, only the detonation velocity.

${ }^{3}$ Boundary conditions should not affect the burn time solution.
} 


\subsection{Mesh}

Region Boundaries ${ }^{4}$ :

2D: $r_{\min }=0.0 \mathrm{~cm}, \theta_{\min }=-\pi / 2$ radians; $r_{\max }=10.0 \mathrm{~cm}, \theta_{\max }=\pi / 2$ radians

3D: $r_{\min }=0.0 \mathrm{~cm}, \theta_{\min }=0.0$ radians, $\phi_{\min }=0.0$ radians; $r_{\max }=10.0 \mathrm{~cm}, \theta_{\max }=\pi / 2$ radians, $\phi_{\max }=\pi$ radians

Mesh Resolutions:

2D Polar:

$$
\begin{aligned}
& N_{r}=40,80,160,320 ; \Delta r=0.25,0.125,0.0625,0.03125 \mathrm{~cm} \\
& N_{\theta}=64,128,256,512 ; \Delta \theta=\pi / 64, \pi / 128, \pi / 256, \pi / 512 \text { radians }
\end{aligned}
$$

3D Spherical:

$$
\begin{aligned}
& N_{r}=40,80,160 ; \Delta r=0.25,0.125,0.0625 \mathrm{~cm} \\
& N_{\theta}=8,16,32 ; \Delta \theta=\pi / 16, \pi / 32, \pi / 64 \text { radians } \\
& N_{\phi}=64,128,256 ; \Delta \phi=\pi / 64, \pi / 128, \pi / 256 \text { radians }
\end{aligned}
$$

\subsection{Output}

In ASCII comma- or space-delimited format ${ }^{5}$, to include:

1. Burn time as a function of position.

\subsection{Comparison Domain}

2D: $(r, \theta) \in[0.0,10.0] \times[-\pi / 2, \pi / 2] 3 \mathrm{D}:(r, \theta, \phi) \in[0.0,10.0] \times[0.0, \pi / 2] \times[0.0, \pi]$

No simulation time steps are necessary for this calculation, since the burn times are calculated at the beginning of a run. One time step may be necessary in a particular code to obtain the desired output.

\footnotetext{
${ }^{4} r$ represents the polar radius in $2 \mathrm{D}$ and the spherical radius in 3D. $\theta$ represents the azimuthal angle in both $2 \mathrm{D}$ and $3 \mathrm{D}$. $\phi$ represents the polar angle in 3D.

${ }^{5}$ Sample output available upon request.
} 


\subsection{Exact Solution}

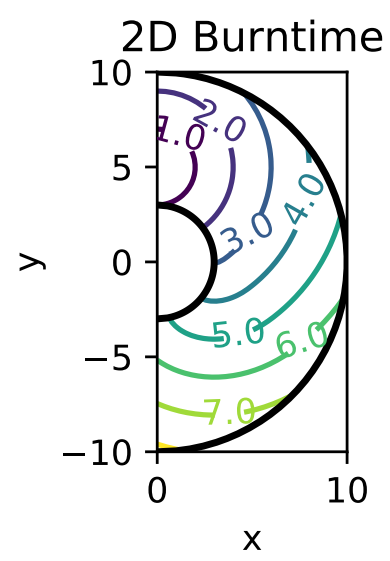

3D Burntime, theta $=0$ plane

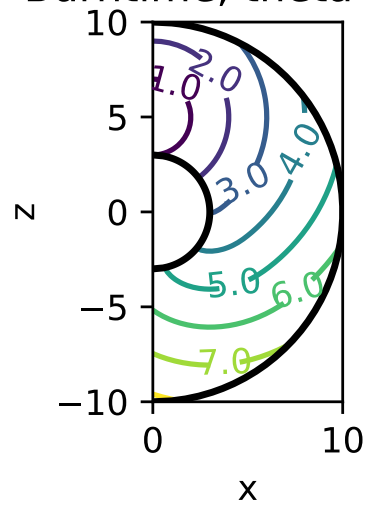

3D Burntime, theta $=\mathrm{pi} / 2$ plane
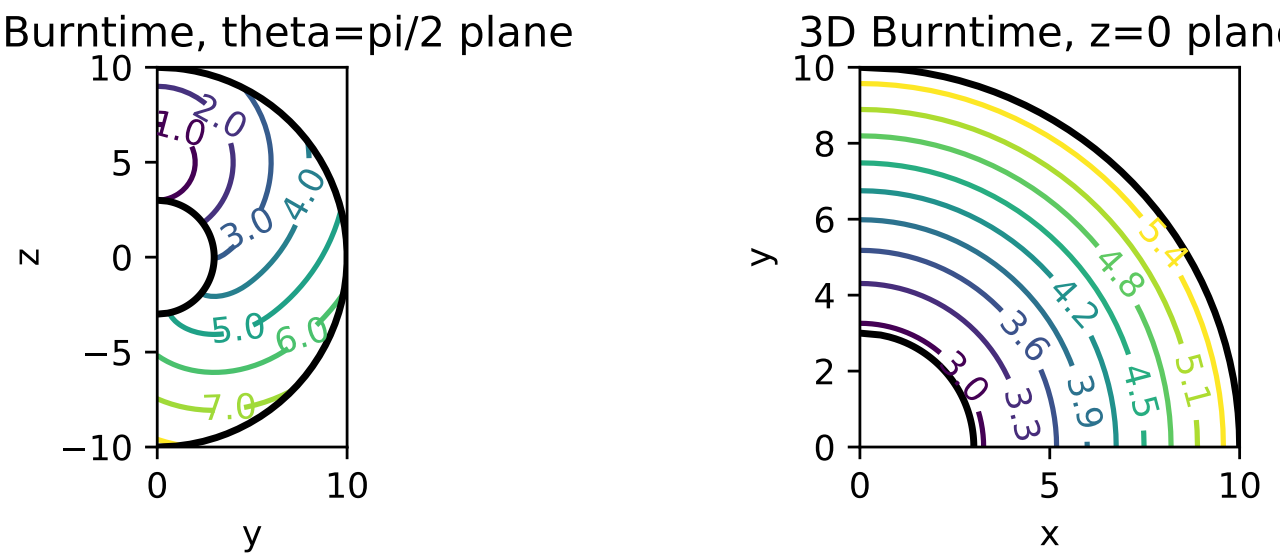


\subsection{Description}

The Kidder Guassian Density problem is a spherically symmetric configuration that invovles the smooth, adiabatic compression follwed by expansion of a compressible gas. The initial state of the inviscid, polytropic gas must be assigned numerically. This problem tests a code's ability to resolve smooth adiabatic flow from compression, through to stagnation, to expansion.

The Kidder problem was first published by Kidder in in 1974 [Kidder]. Ramsey, et al. derives the general solution for this class of Gaussian density problems, provides computational results and analysis along with practical advice on setting up this problem [Ramsey]. See also [Kamm].
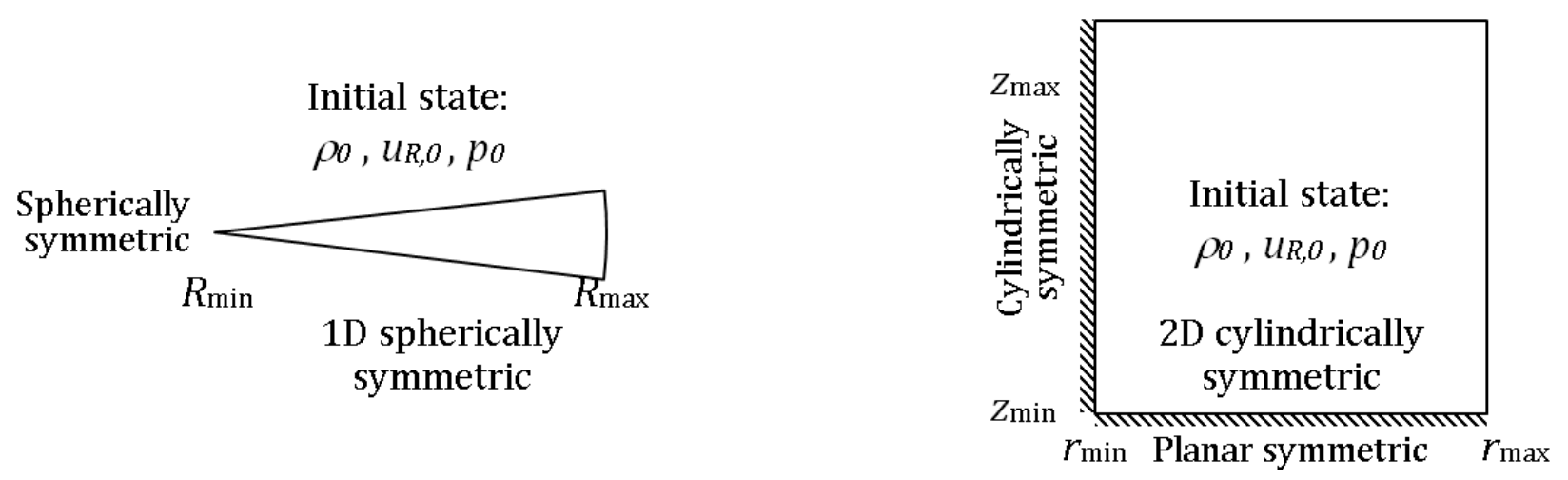

Fig. 11.1: Initial 1D and 2D configurations of the Kidder Gaussian Density problem.

\subsection{Solution}

Under the assumptions of a polytropic gas, with $p=(\gamma-1) \rho e$, and given the Initial and Boundary Conditions described below. The Kidder Gaussian Density problem admits the closed-form solution shown in the Solution table. The corresponding problem parameters are given in Run parameters table.

Table 11.1: Solution to the Kidder Problem.

\begin{tabular}{|l|l|}
\hline Physical Field & Analytic Solution \\
\hline$\rho\left[\mathrm{g} / \mathrm{cm}^{3}\right]$ & $2\left(1+t^{2}\right)^{-3 / 2} \exp \left[-r^{2} /\left(1+t^{2}\right)\right]$ \\
\hline$u[\mathrm{~cm} / \mathrm{s}]$ & $r t /\left(1+t^{2}\right)$ \\
\hline$e[\mathrm{erg} / \mathrm{g}]$ & $(3 / 4) /\left(1+t^{2}\right)$ \\
\hline
\end{tabular}




\subsection{Problem Parameters}

The following table gives the constant parameters for Kidder problem. The Initial values table contains the initial distribution of the physical fields, found by substituting $t=-1$ into the exact solution given in the Solution table above

Table 11.2: Run parameters for the Kidder Problem.

\begin{tabular}{|l||l||l||l|}
\hline$t_{\text {init }}$ & $t_{\text {fin }, 0}$ & $t_{\text {fin }, 1}$ & $\gamma$ \\
\hline$[\mathbf{s}]$ & {$[\mathrm{s}]$} & {$[\mathbf{s}]$} & {$[-]$} \\
\hline 1 & 0 & 1 & $5 / 3$ \\
\hline
\end{tabular}

\subsection{Initial Conditions}

Spatially dependent material state over the entire domain, determined by the anlytic solution. Substituting $t=-1$ into the solutions shown in the solution gives the table below. Note that initial density, velocity and energy are specified as a function of radius over the range, $r \in[0,3]$. The initial pressure profile in the table was obtained from the solutions for $\gamma, \rho$ and $e$ in solution using the formula $p=(\gamma-1) \rho e$. Note that this table is redundant, in that the pressure need not be specified, however it was included for completeness and because it is sometimes used to test of consistency of the input deck at the first time step.

Table 11.3: Initial values for the Kidder Problem.

\begin{tabular}{|l|l|}
\hline Physical Field & Initial Distribution \\
\hline$r_{\text {init }}[\mathrm{cm}]$ & $r_{\text {init }} \in[0,3] \mathrm{cm}$ \\
\hline$\rho_{\text {init }}\left[\mathrm{g} / \mathrm{cm}^{3}\right]$ & $\exp \left[-r^{2} / 2\right]$ \\
\hline$u_{\text {init }}[\mathrm{cm} / \mathrm{s}]$ & $-r / 2$ \\
\hline$e_{\text {init }}[\mathrm{erg} / \mathrm{g}]$ & $3 / 8$ (Uniform) \\
\hline$p_{\text {init }}[\mathrm{erg} / \mathrm{g}]$ & $(1 / 4) \exp \left[-r^{2} / 2\right]$ \\
\hline
\end{tabular}

\subsection{Boundary Conditions}

The problem is to be run so that any spurious waves generated by boundary conditions do not affect the solution on the Comparison Domain defined below. Possible boundary conditions are:

Inner: $R_{\min }, r_{\min }, z_{\min }:$ Symmetric

Outer: $R_{\max }, r_{\max }, z_{\max }$ : Constant in time

\subsection{Mesh}

Region Boundaries ${ }^{1}$ :

$R_{\min }=0.0, R_{\max }=3.0 \mathrm{~cm} ;$ in $1 \mathrm{D}$

$(r, z)_{\min }=0.0,(r, z)_{\max }=3.0 \mathrm{~cm} ;$ in $2 \mathrm{D}$,

Mesh Resolutions:

\footnotetext{
${ }^{1}$ Here, $R=$ spherical radial coordinate, with $R=r^{2}+z^{2}$, where $r=$ cylindrical radial coordinate.
} 
1D spherical: $N_{R}=60,120,240,480,960 ; \Delta_{R}=0.5,0.025,0.0125,0.00625,0.003125$;

2D cylindrical: $N_{r}=N_{z}=60,120,240,480,960 ; \Delta_{r}=\Delta_{z}=0.5,0.025,0.0125,0.00625,0.003125$

\subsection{Output}

In ASCII comma- or space-delimited format, ${ }^{2}$ to include:

1. Values of density, velocity, pressure, SIE as a function of position at $t_{0}, t_{\mathrm{fin}, 0}$ and $t_{\mathrm{fin}, 1}$.

2. Entire mesh total energy, kinetic energy, internal energy as a function of time.

\subsection{Comparison Domain}

1D: $R \in[0,1]$

$2 \mathrm{D}:(r, z) \in[0,1] \times[0,1]$

${ }^{2}$ Sample output available upon request. 


\subsection{Description}

These 1D Riemann Problems are finite-dimensional restrictions of mathematically ideal, infinite domain plasmas. They have two materials in Cartesian geometry separated by a massless interface. At $t=0$, the plasmas are uniform and in pressure equilibrium. The removal of the interface leads to the evolution of the self-similar solution, consisting of a front of dense plasma diffusing into lighter plasma, and the lighter plasma diffusing throughout the dense material.

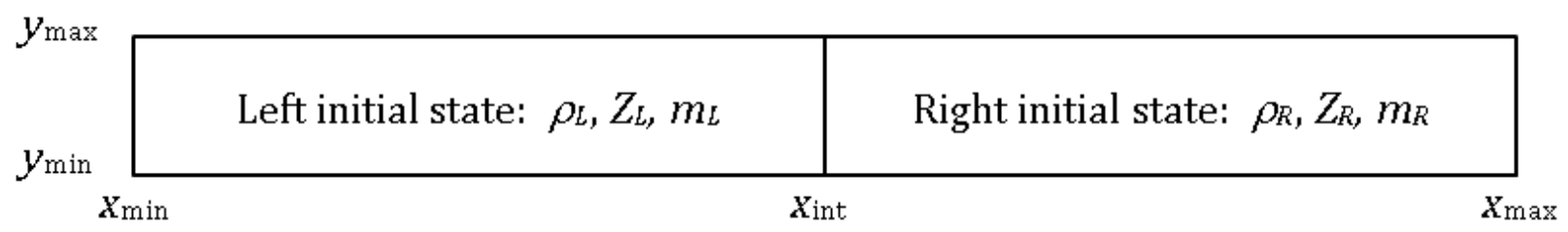

Fig. 12.1: Initial geometry of Cartesian mass diffusion problems.

\subsection{Problem Parameters}

The parameters in the tables below correspond to the following tests [Molvig],

1. Gold Plasma: ICF plasma conditions relevant to heavy metal pushers used in double shell capsule designs. These conditions were those typically condisdered during the theoretical development of the diffusion model in Ref [Molvig].

2. Aluminum Plasma: ICF relevant conditions used to verify the theory against kinetic simulations (particle-incell method with a binary collision model). This case also tests the model at different plasma conditions.

Table 12.1: Global parameters for the 1D mass-diffusion problems

\begin{tabular}{|l|l|l|l|l|l|}
\hline Test & $x_{\text {int }}$ & $t_{\text {fin }}$ & $u$ & $\mathrm{P}$ & $\mathrm{T}$ \\
\hline & {$[\mathrm{cm}]$} & {$[\mathrm{s}]$} & {$[\mathrm{cm} / \mathrm{s}]$} & {$\left[\mathrm{erg} / \mathrm{cm}^{3}\right]$} & {$[\mathrm{eV}]$} \\
\hline 1 & 0.0050 & $2 \times 10^{-9}$ & 0 & $2.41 \times 10^{15}$ & 5000 \\
\hline 2 & 0.0050 & $6 \times 10^{-9}$ & 0 & $3.74 \times 10^{16}$ & 4000 \\
\hline
\end{tabular}


Table 12.2: Parameters for left and right plasma sections

\begin{tabular}{|l|l|l|l|l|l|l|}
\hline Test & $\rho_{\mathrm{L}}$ & $\rho_{\mathrm{R}}$ & $Z_{\mathrm{L}}$ & $Z_{\mathrm{R}}$ & $m_{\mathrm{L}}$ & $m_{\mathrm{R}}$ \\
\hline & {$\left[\mathrm{g} / \mathrm{cm}^{3}\right]$} & {$\left[\mathrm{g} / \mathrm{cm}^{3}\right]$} & {$[\mathrm{e}]$} & {$[\mathrm{e}]$} & {$[\mathrm{amu}]$} & {$[\mathrm{amu}]$} \\
\hline 1 & 1.26 & 0.5 & 77 & 1 & 197 & 2 \\
\hline 2 & 18.7 & 9.7 & 13 & 1 & 27 & 2 \\
\hline
\end{tabular}

\subsection{Initial conditions}

The material velocity, pressure and temperature are uniform and constant across the initial interface $x=x_{\text {int }}$. The density is uniform and constant on each side of the interface. Other states are related through the polytropic equation of state for a perfect gase: $p=(\gamma-1) \rho e$, where, $\gamma=5 / 3$, is the (constant) ratio of specific heats.

\subsection{Boundary conditions}

Left/right: $x_{\min }, x_{\max }:$ Constant in time

\subsection{Mesh}

Region Boundaries:

For all problems, $x_{\min }=0.0000, x_{\max }=+0.0100 \mathrm{~cm}$.

Mesh Resolutions:

1D: $N_{x}=25,50,100,200,400 ; \Delta_{x}=0.000400,0.000200,0.000100,0.000050,0.000025 \mathrm{~cm}$

\subsection{Output}

In ASCII comma- or space-delimited format ${ }^{1}$, to include:

1. Values of species density, velocity and pressure as a function of position at $t_{0}$ and $t_{\text {fin }}$.

2. Entire mesh total energy, kinetic energy, internal energy as a function of time.

\subsection{Comparison Domain}

1D: $x \in[0.0000,0.0100]$

\subsection{Test problem 1: results at $t_{\text {fin }}=2 \times 10^{-9} \mathbf{s}$}

This figure shows the interpenetration of a gold plasma (black line) into a deuterium plasma (red line) at $t=2 \times 10^{-9} \mathrm{~s}$, and corresponds to Fig. 2 of Ref. [Molvig]. The initial deuterium density profile is outlined (dotted red line), as well

\footnotetext{
${ }^{1}$ Sample output available upon request.
} 


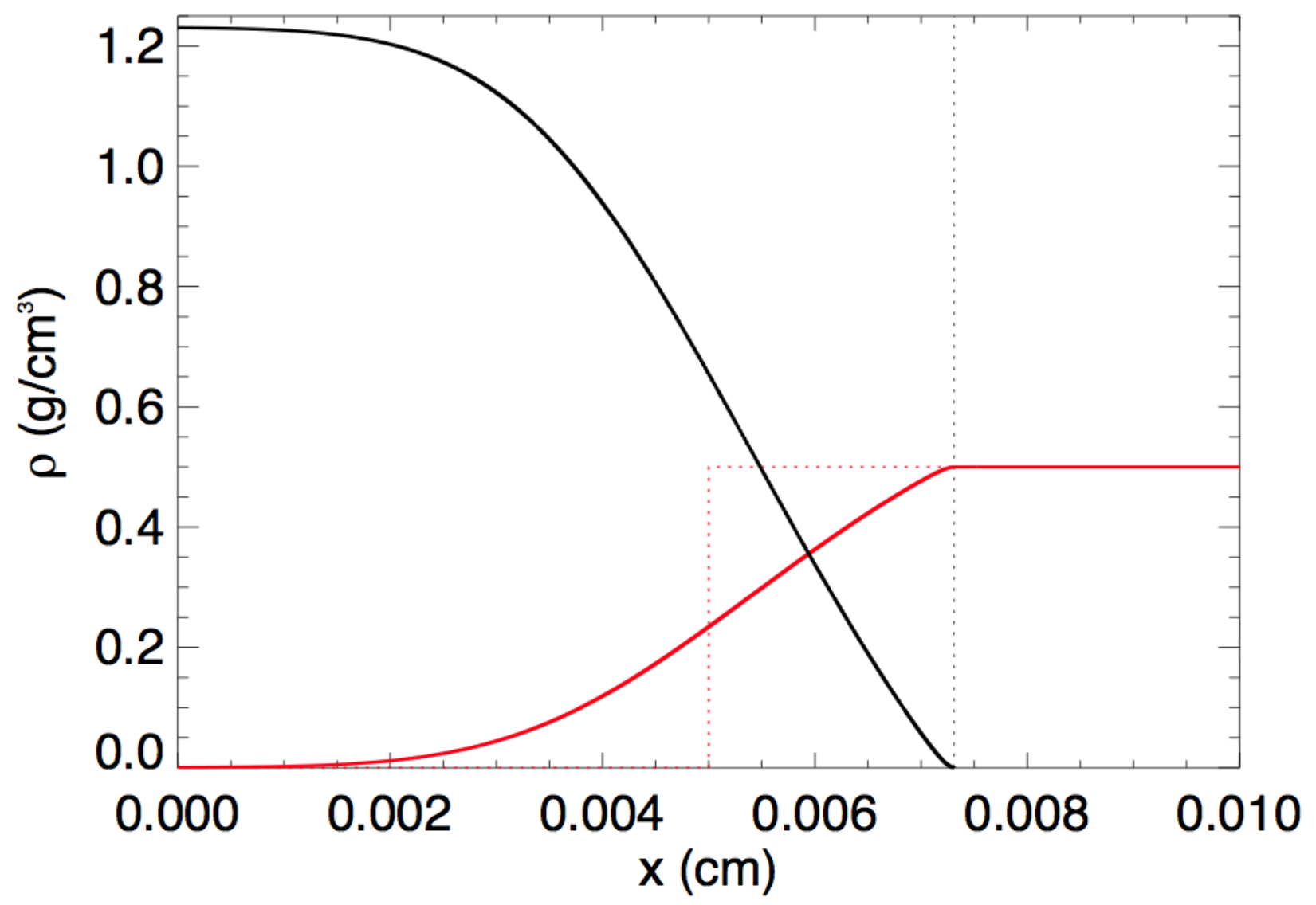

as the final gold front position (dotted black line). This test case produces a mixed region extending from $x \approx 0.0020$ $\mathrm{cm}$ to $x \approx 0.0073 \mathrm{~cm}$

\subsection{Test problem 2: results at $t_{\text {fin }}=6 \times 10^{-9} \mathbf{s}$}

This figure shows the interpenetration of an aluminum plasma (black line) into a deuterium plasma (red line) at $t=6 \times 10^{-9} \mathrm{~s}$, and corresponds to Fig. 4 of Ref. [Molvig]. The initial deuterium density profile is outlined (dotted red line), as well as the final aluminum front position (dotted black line). This test case produces a mixed region extending from $x \approx 0.0020 \mathrm{~cm}$ to $x \approx 0.0073 \mathrm{~cm}$ 


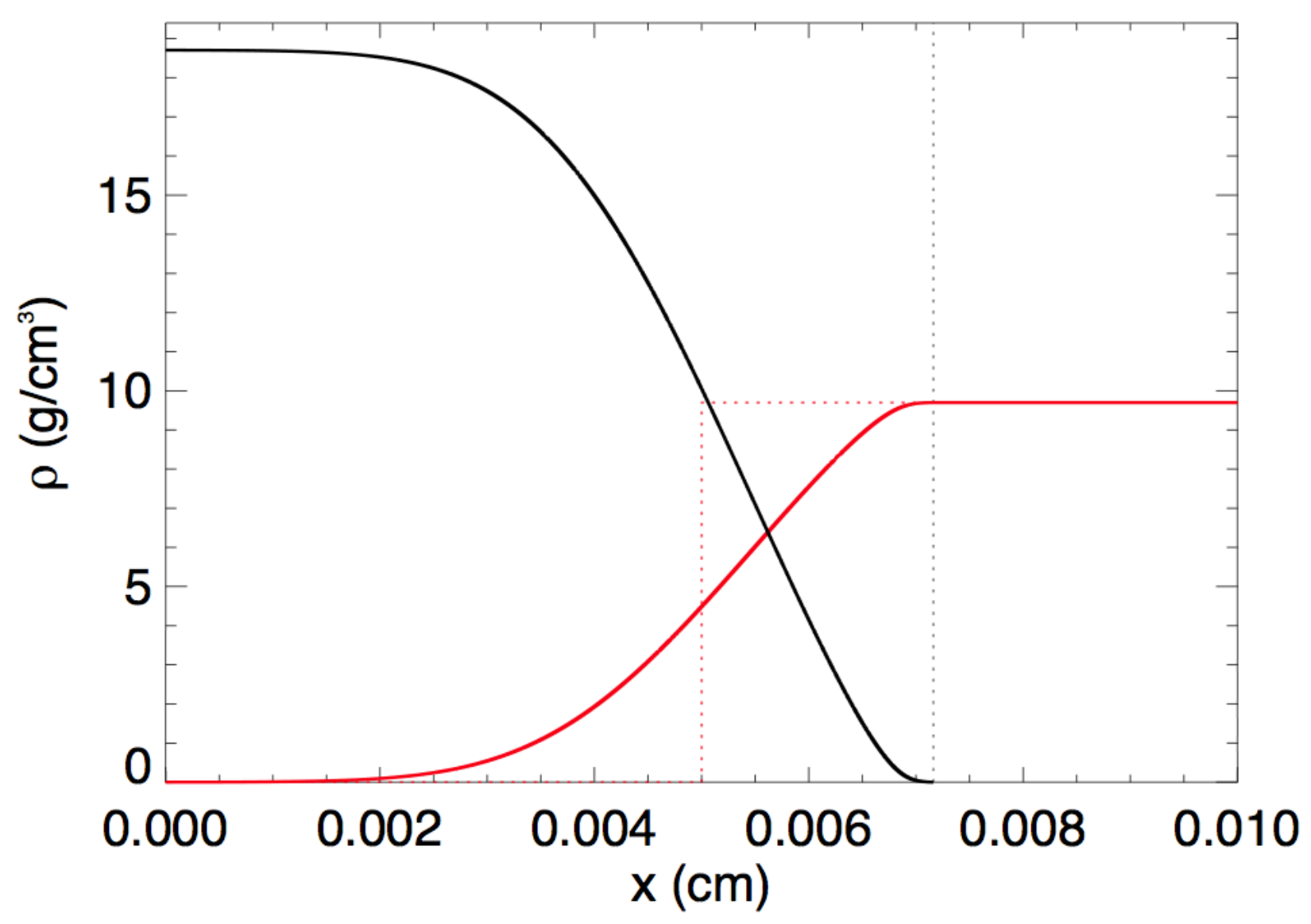




\subsection{Description}

1D Riemann Problems are finite-dimensional restrictions of mathematically ideal, infinite domain shock tube experiments. They are in Cartesian geometry with two materials separated by a massless interface. At $t=0$, the states are constant and uniform. The removal of the interface leads to the evolution of the self-similar solution, consisting of some combination of shock, contact, and rarefaction waves.

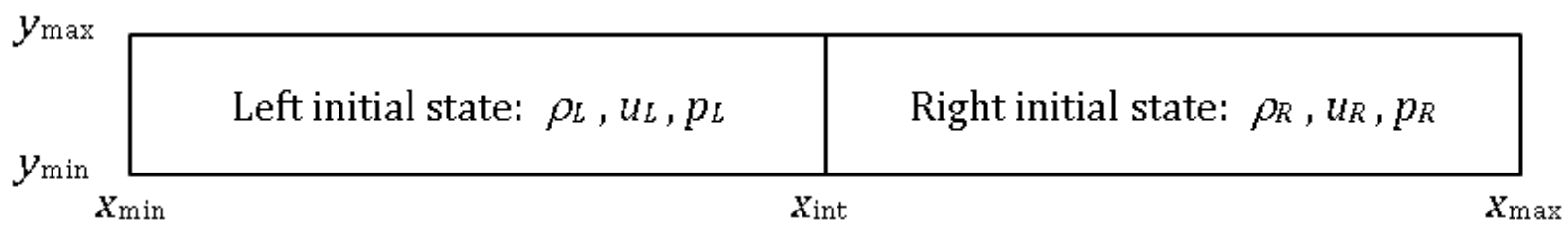

Fig. 13.1: Initial geometry of Cartesian Riemann problems.

\subsection{Problem Parameters}

The parameters in the Global and Left and Right parameters tables, correspond to the following tests [Kamm],

1. Sod shock tube: The canonical shock tube problem with rarefaction-contact-shock structure; while not a challenging problem, it quickly identifies algorithmic problems resolving basic wave structure.

2. Einfeldt (or 1-2-3) problem: Consists of two strong rarefaction waves, with a near-vacuum between them; methods that conserve total energy might show internal energy errors for this problem.

3. Stationary contact problem: Consists of a strong shock wave moving to the right, a stationary contact, and a strong rarefaction moving to the left; it is based on the left part of the well-known Woodward-Colella problem, but with the velocity shifted to make the contact stationary, and tests an algorithm's dissipation by how much the contact is smeared.

4. Slow shock problem: Consists of a Mach 3 shock wave moving slowly to the right; some numerical methods exhibit unphysical oscillations behind the shock.

5. Shock-contact-shock problem: When two shocks separate from the initial state, with a contact between them, errors are producted in all fields, and this problem tests how well an algorithm deals with thos errors; this is similar to the planar Noh problem but with weaker shocks.

6. LeBlanc problem: A strong shock, strong rarefaction version of the basic rarefaction-contact-shock problem; it is a good test of a method's robustness. 
7. Test 7: Explosive products expanding into air (Aslam, 2015), $\rho_{L}=1.84, p_{L}=0.37, u_{L}=0 ., \gamma_{L}=2.851$, and $\rho_{R}=1 . e-3, p_{R}=1 . e-6, u_{R}=0 ., \gamma_{R}=1.4$. Problem extent is $[-1,1]$ with initial interface at $x=0$.

Table 13.1: Global parameters for the six Riemann problems.

\begin{tabular}{|l|l|l|l|}
\hline Test & $x_{\text {int }}$ & $t_{\text {fin }}$ & $\gamma$ \\
\hline & {$[\mathrm{cm}]$} & {$[\mathrm{s}]$} & {$[-]$} \\
\hline 1 & 0.5 & 0.25 & $7 / 5=1.4$ \\
\hline 2 & 0.5 & 0.15 & $7 / 5$ \\
\hline 3 & 0.9 & 0.012 & $7 / 5$ \\
\hline 4 & 0.5 & 1.0 & $7 / 5$ \\
\hline 5 & 0.5 & 0.3 & $7 / 5$ \\
\hline 6 & 0.5 & 0.5 & $5 / 3=1.66667$ \\
\hline
\end{tabular}

Table 13.2: Parameters for left and right shock tube sections.

\begin{tabular}{|l|l|l|l|l|l|l|}
\hline Test & $\rho_{L}$ & $u_{L}$ & $p_{L}$ & $\rho_{R}$ & $u_{R}$ & $p_{R}$ \\
\hline & {$\left[\mathrm{g} / \mathrm{cm}^{3}\right]$} & {$[\mathrm{cm} / \mathrm{s}]$} & {$\left[\mathrm{dyn} / \mathrm{cm}^{2}\right]$} & {$\left[\mathrm{g} / \mathrm{cm}^{3}\right]$} & {$[\mathrm{cm} / \mathrm{s}]$} & {$\left[\mathrm{dyn} / \mathrm{cm}^{2}\right]$} \\
\hline 1 & 1.0 & 0.0 & 1.0 & 0.125 & 0.0 & 0.1 \\
\hline 2 & 1.0 & -2.0 & 0.4 & 1.0 & 2.0 & 0.4 \\
\hline 3 & 1.0 & -19.59745 & $1 \times 10^{3}$ & 1.0 & -19.59745 & $1 \times 10^{-2}$ \\
\hline 4 & 3.857143 & -0.810631 & 10.33333 & 1.0 & -3.44 & 1.0 \\
\hline 5 & 1.0 & 0.5 & 1.0 & 1.25 & -0.5 & 1.0 \\
\hline 6 & 1.0 & 0.0 & $(2 / 3) \times 10^{-1}$ & $1 \times 10^{-2}$ & 0.0 & $(2 / 3) \times 10^{-10}$ \\
\hline
\end{tabular}

\subsection{Initial conditions}

Uniform and constant material density, pressure, and velocity on each side of the initial interface ( $\mathrm{x}=\mathrm{xint})$. Other states are related through the polytropic EOS: $p=(\gamma-1) \rho e$, where, $\gamma$ is the (constant) ratio of specific heats.

\subsection{Boundary conditions}

Left/right: $x_{\min }, x_{\max }$ : Constant in time

Top/Bottom: $y_{\min }, y_{\max }$ : Reflective

\subsection{Mesh}

For all problems, $x_{\min }=0.0$ and $x_{\max }=1.0 \mathrm{~cm}$; in $2 \mathrm{D}, y_{\min }=0.0, y_{\max }=0.2 \mathrm{~cm}$.

1D: $N_{x}=50,100,200,400,800$

2D: $\left(N_{x}, N_{y}\right)=(50,10),(100,20),(200,40),(400,80),(800,160)$

\subsection{Output}

In ASCII comma- or space-delimited format ${ }^{1}$, to include:

\footnotetext{
${ }^{1}$ Sample output available upon request.
} 
1. Values of density, velocity, pressure, SIE as a function of position at $t_{0}$ and $t_{\text {fin }}$.

2. Entire mesh total energy, kinetic energy, internal energy as a function of time.

\subsection{Comparison Domain}

1D: $x \in[0,1]$

2D: $(x, y) \in[0,1] \times[0,0.2]$

\subsection{Extension of the Computational Domain for Moving Walls}

The computational domain $[0,1]$ requires an extension to a larger mesh when the wall at either end, $x_{\min }=0$ or $x_{\max }=1$, is moving, i.e. cases 2 through 5 in Global parameters and Left and Right parameters tables. We denote the expanded domain by $\left[x_{L}, x_{R}\right]$ with $x_{L}<x_{\min }<x_{\text {int }}<x_{\max }<x_{R}$. An adequate choice for numerical implementations of Problems 2-5 is $x_{L}=-1$ and $x_{R}=2$. Implementation details for specific codes are shown in the Numerical Setup page.

\subsection{Exact Solutions at $t_{\text {fin }}$}

The exact solutions at $t_{\text {fin }}$ for all six Riemann Problems are plotted below: 


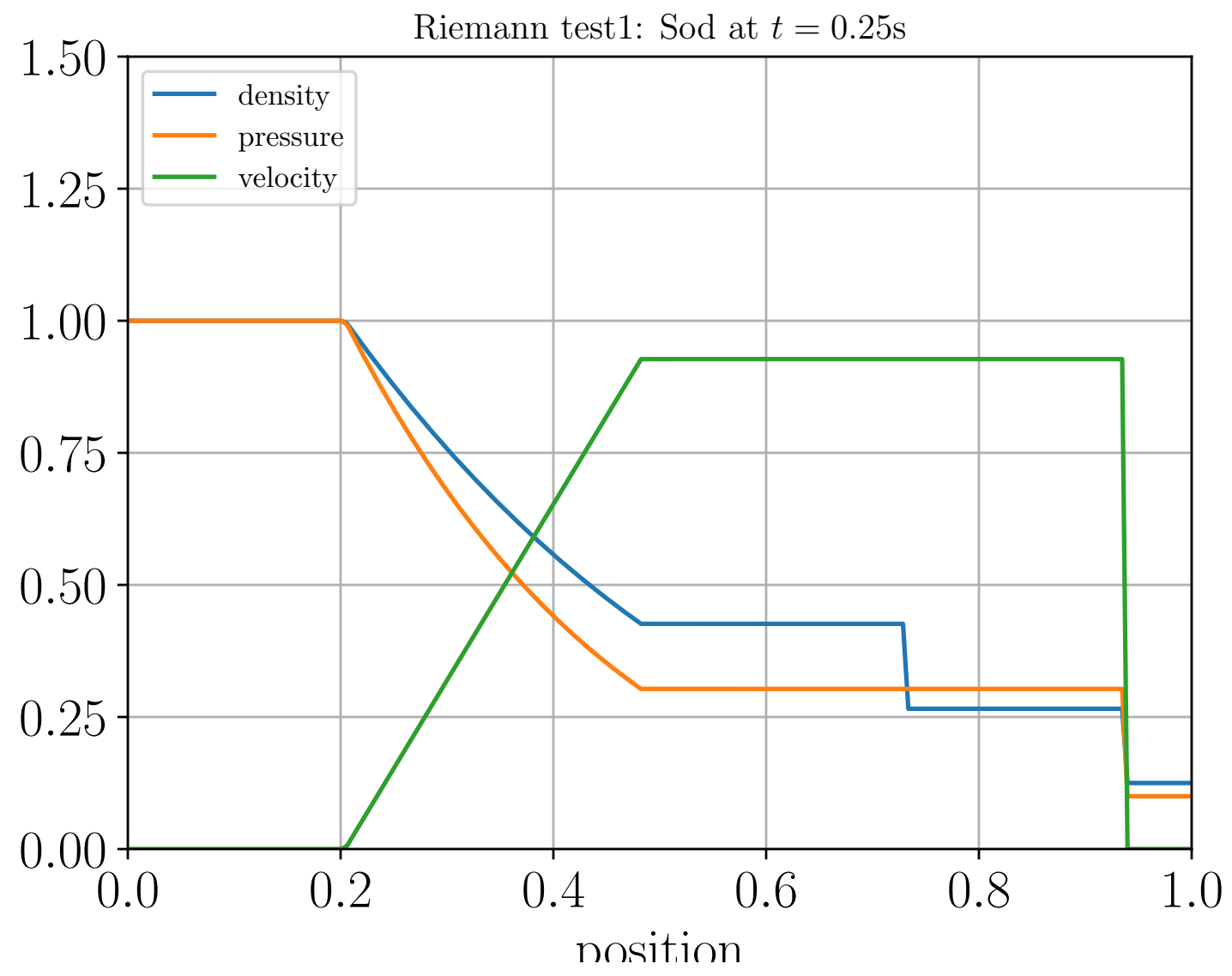




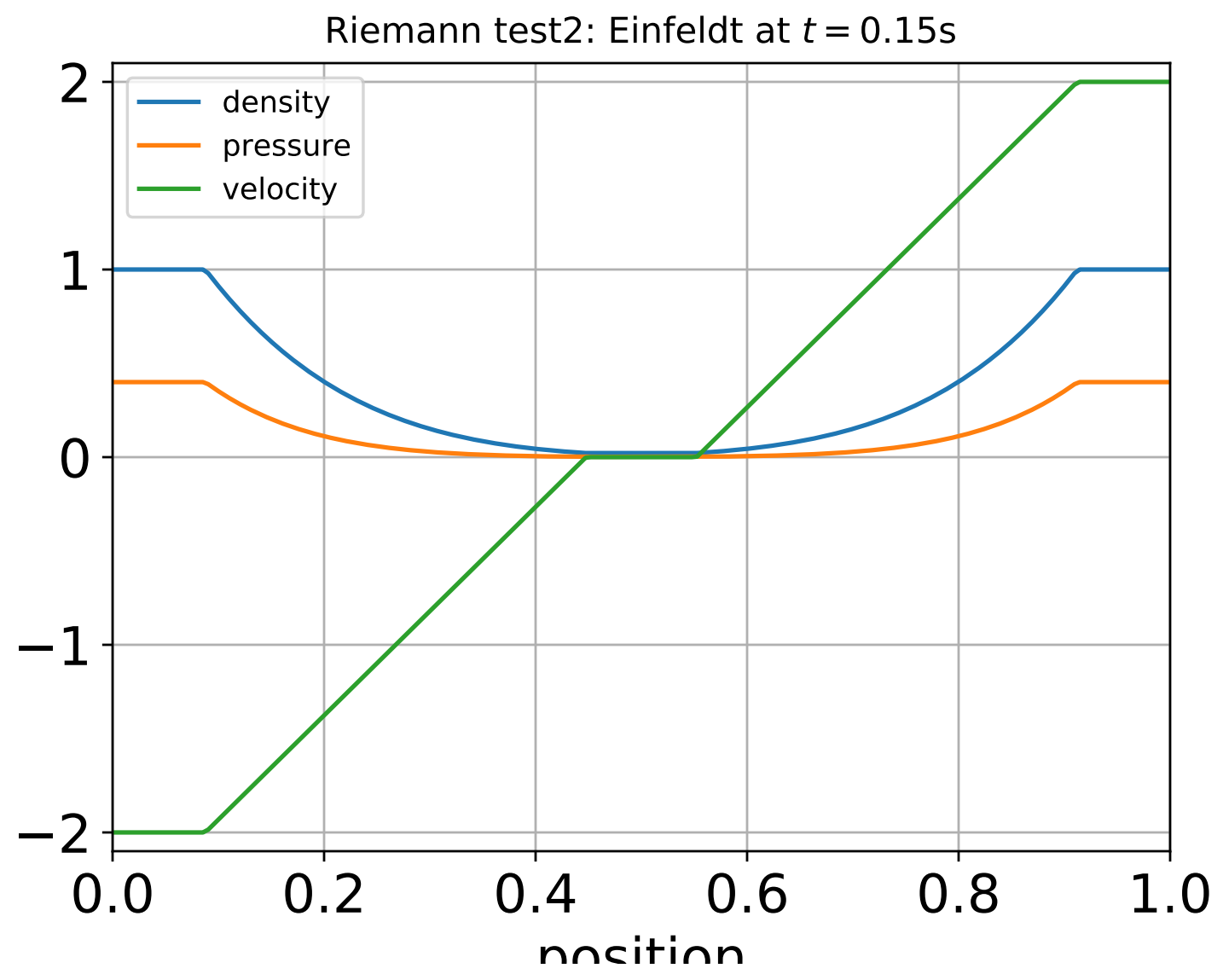




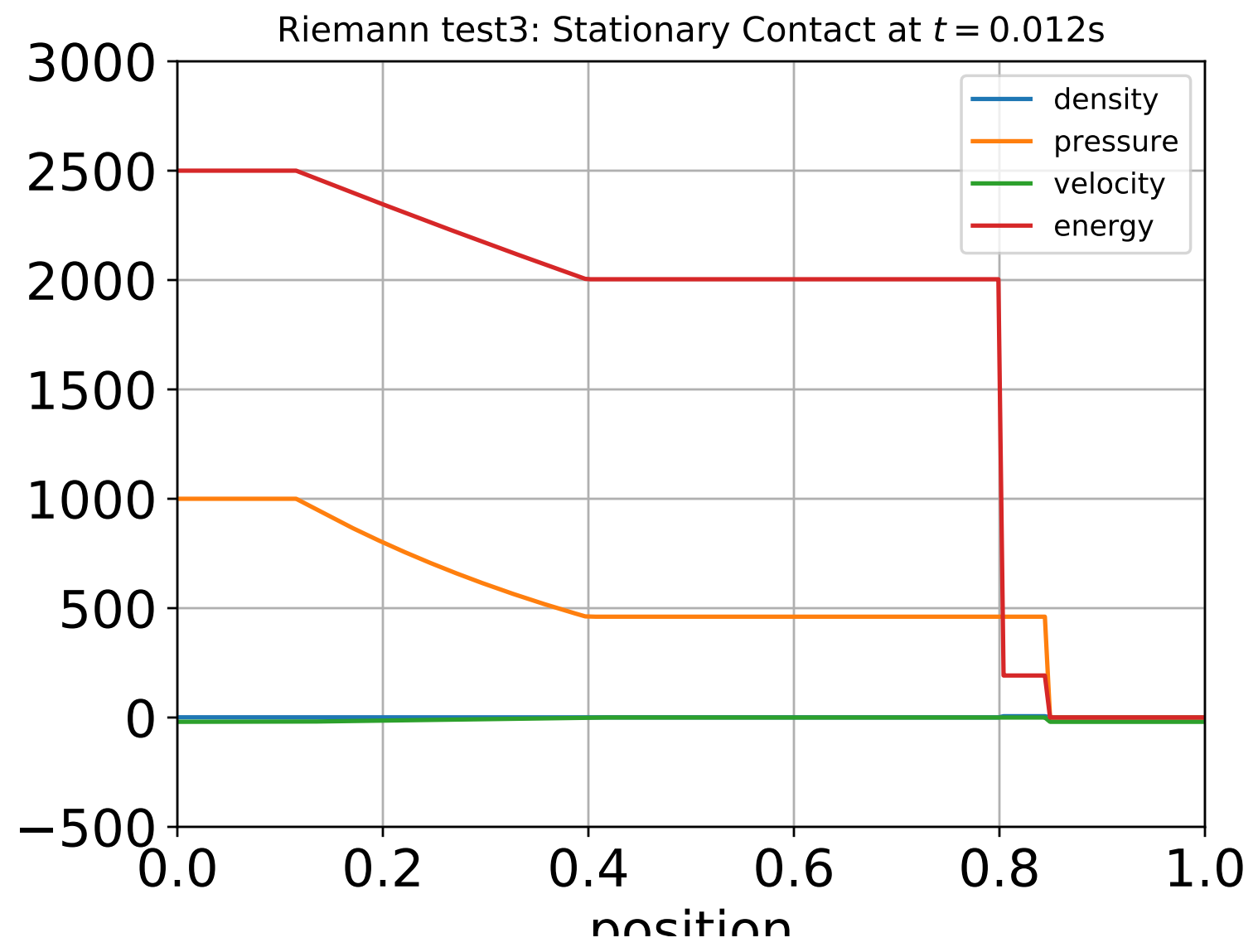




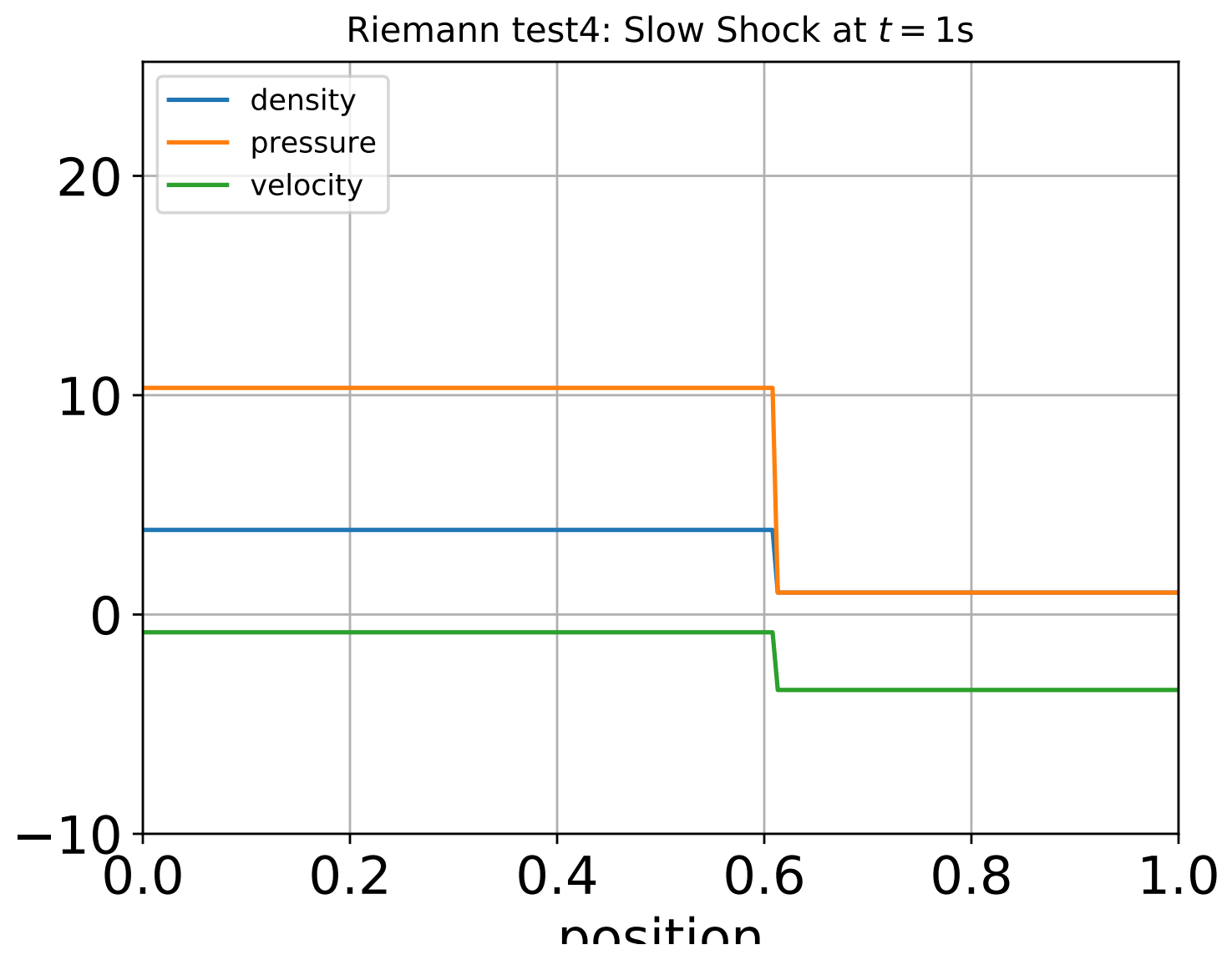




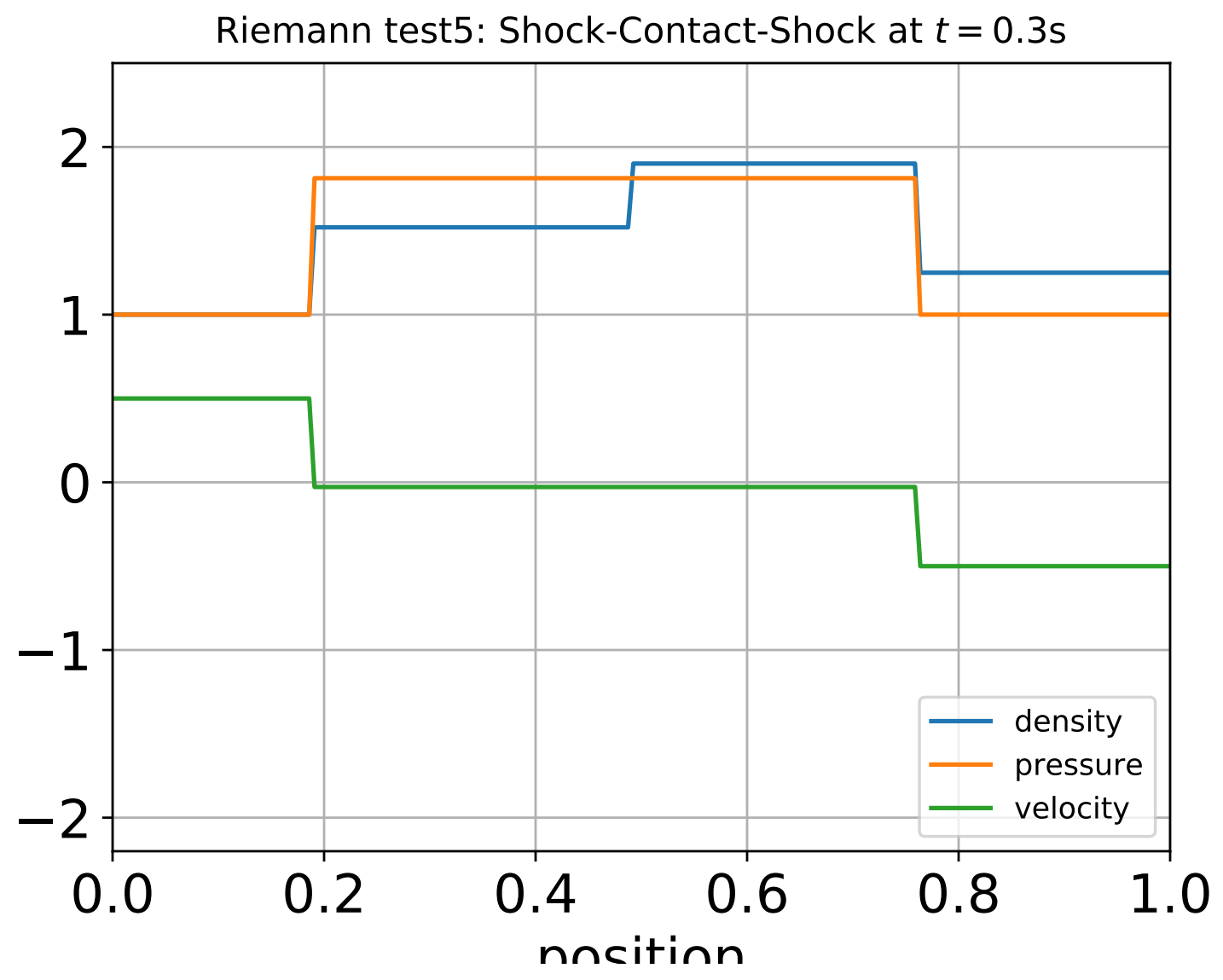




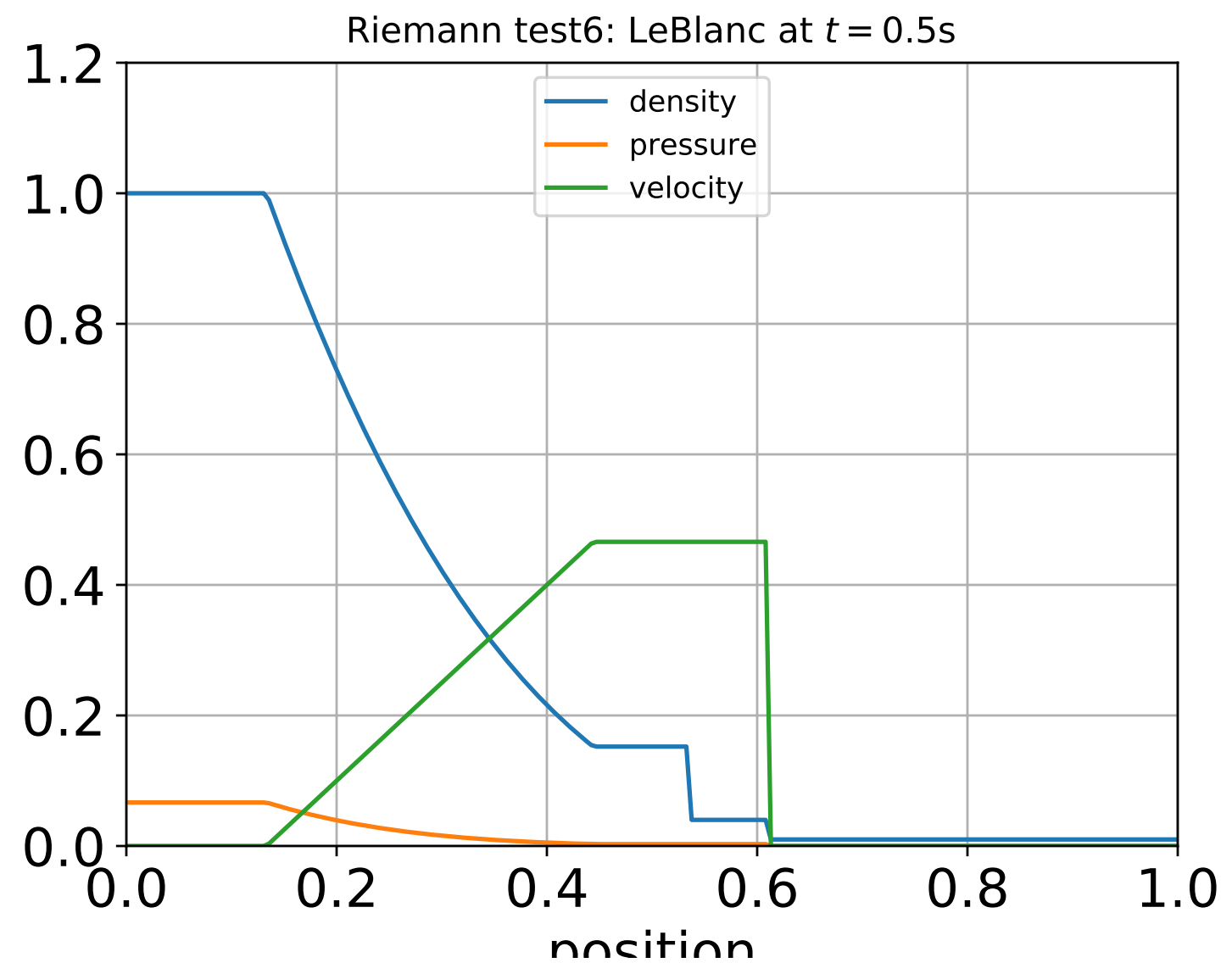




\section{NUMERICAL SETUP FOR 1D RIEMANN PROBLEMS}

The 1D Riemann Problems require extention to a larger mesh to allow for moving walls, as is discussed in the Riemann Problems description page.

\subsection{Extension of the Computational Domain for Moving Walls}

In the 1D Riemann Problems, the computational domain [0,1] requires an extension to a larger mesh when one of the walls at either end, $x_{\min }=0$ or $x_{\max }=1$, is moving, i.e. cases 2 through 5 of Global parameters and Left and Right parameters tables. We denote the expanded domain by $\left[x_{L}, x_{R}\right]$ with $x_{L}<x_{\min }<x_{\text {int }}<x_{\max }<x_{R}$. Except for test problem 4 , the choice $x_{L}=-1$ and $x_{R}=2$ is adequate.

\subsection{Eulerian Code}

We place $n p t s=50,100,200,400,800$ points in the computational domain $[0,1]$. In problems 2 , 3, and 5, we account for the wall's motion by extending the computational domain to $[-1,2]$, and for the slow shock of problem 4 we extend the grid to $[-2,3]$. We place $n p t s$ points in each extension of unit length, e.g. $[-1,0]$ and $[1,2]$. For the grid $[-1,2]$ this gives a total of $n p t s 2=3 \cdot$ npts points in $[-1,2]$, and for $[-2,3]$ this gives $n p t s 2=5 \cdot$ npts points. Problem 4 also imposes freeze conditions on either extreme of the mesh $[-2,3]$.

\subsection{Lagrangian Code}

In problems 2 through 5 , we account for the wall's motion by extending the computational domain from $\left[x_{\min }=\right.$ $\left.0, x_{\max }=1\right]$ to the larger domain $\left[x_{L}=-1, x_{R}=2\right]$. The domain is partitioned into left and right regions by the membrane at location $x_{\text {int }}$. The number of points to in the left and right regions of the computational zone $\left[x_{\min }, x_{\max }\right]$ is

$$
\begin{aligned}
& N_{\mathrm{L}}=\left[\frac{x_{\mathrm{int}}-x_{\mathrm{min}}}{x_{\max }-x_{\min }} \cdot N_{\mathrm{K}}\right] \\
& N_{\mathrm{R}}=\left[\frac{x_{\max }-x_{\mathrm{int}}}{x_{\max }-x_{\min }} \cdot N_{\mathrm{K}}\right],
\end{aligned}
$$

where the function $[x]$ denotes the greatest integer less than or equal to $x$. The number of points between $x_{\mathrm{L}}{ }^{6} a n d$ : math: ' $x_{\min }$ is denoted by $x_{\mathrm{I}}$, while between $x_{\mathrm{R}}$ and $x_{\max }$ is denoted by $x_{\mathrm{J}}$. The implementation chooses

$$
\begin{aligned}
& N_{\mathrm{I}}=\left[\frac{x_{\mathrm{L}}-x_{\min }}{x_{\max }-x_{\min }} \cdot N_{\mathrm{K}}\right] \\
& N_{\mathrm{J}}=\left[\frac{x_{\mathrm{R}}-x_{\max }}{x_{\max }-x_{\min }} \cdot N_{\mathrm{K}}\right],
\end{aligned}
$$


giving the total number of points in the left and right extended regions as

$$
\begin{aligned}
& N_{\mathrm{L}}^{\text {ext }}=N_{\mathrm{L}}+N_{\mathrm{I}} \\
& N_{\mathrm{R}}^{\text {ext }}=N_{\mathrm{R}}+N_{\mathrm{J}} .
\end{aligned}
$$




\subsection{Description}

The Mader Problem is a one-dimensional piston-driven detonation wave with a trailing polytropic rarefaction, in which a slab of high explosive (HE) is initiated on one side and a detonation wave propagates to the other side. The Mader Problem tests a code's ability to compute the evolution of the rarefaction behind the burn front and the CJ state at the detonation front, assuming the detonation wave propagates through a one-dimensional compressible gas.

The Mader Problem is a special case of the detonation wave solution given on page 24 of Ficket and Davis [Fickett]. A detonation wave is driven by a piston moving in the $+x$ direction in a one-dimensional $5 \mathrm{~cm}$ slab of gamma-law gas. A rarefaction (i.e., a Taylor wave) follows the detonation front. The Mader Problem solution is given here in the rest frame of the pistion. The head of the rarefaction is at the detnoation front and the tail is half-way between the front and the piston. For detailed analysis see Timmes, et al, [Timmes], and Kirkpatrick, et al [Kirkpatrick], See also [Kamm].

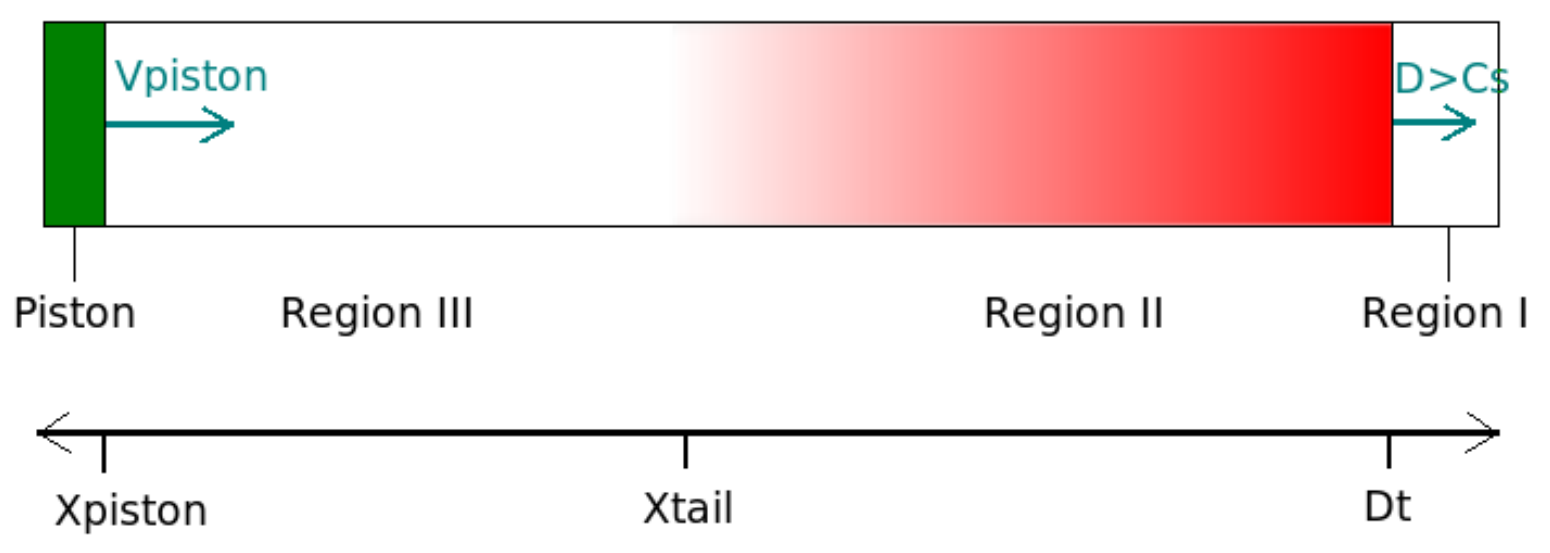

Fig. 15.1: Geometry of the Mader Problem 


\subsection{Problem Parameters}

The following table gives the constant parameters for Mader Problem. The Initial Conditions section describes the initial state of the system. The specific reaction enthalpy, $q=\frac{D_{c j}^{2}}{2\left(\gamma^{2}-1\right)}$, is computed from the values of $D_{c j}$ and $\gamma$.

Table 15.1: Parameters for the Mader

Problem.

\begin{tabular}{|l|l|l|l|}
\hline$t_{\text {fin }}$ & $\gamma$ & $D_{c j}$ & $q$ \\
\hline$[\mu s]$ & {$[-]$} & {$[\mathrm{cm} / \mu \mathrm{s}]$} & {$[\mathrm{erg} / \mathrm{gm}]$} \\
\hline 6.25 & 3 & 0.8 & $4.0 \times 10^{10}$ \\
\hline
\end{tabular}

\subsection{Initial Conditions}

Initial conditions in Region I are given in the table below. This table represents a nominal initial state in Region I correpsonding to the anaylitic solution given here. The rarefaction solution should be somewhat (or entirely) insensitive to the initial pressure and sound speed as long as a simulation code is able to sufficiently approximate the given CJ state directly behind the detonation front, so there may be some freedom to deviate somewhat from this prescription, if necesssary, for running the Mader Problem on individual codes.

Table 15.2: Initial Conditions for the Mader Problem.

\begin{tabular}{|l|l|l|l|}
\hline$v_{0}$ & $\rho_{0}$ & $p_{0}$ & $c_{0}$ \\
\hline$[\mathrm{cm} / \mu \mathrm{s}]$ & {$\left[\mathrm{g} / \mathrm{cm}^{3}\right]$} & {$\left[\mathrm{dyn} / \mathrm{cm}^{2}\right]$} & {$[\mathrm{cm} / \mu \mathrm{s}]$} \\
\hline$-v_{\text {piston }}$ & 1.875 & 0.0 & 0.0 \\
\hline
\end{tabular}

\subsection{Boundary Conditions}

The problem is to be run so that any spurious waves generated by boundary conditions do not affect the solution on the Comparison Domain defined below. Possible boundary conditions are:

Inner: $X_{\min }, Y_{\min }, Z_{\min }:$ Symmetric

Outer: $X_{\max }, Y_{\max }, Z_{\max }$ : Constant in time

\subsection{Mesh}

Region Boundaries:

The Region is a slab geometry so widths in the $\mathrm{Y}$ and $\mathrm{Z}$ directions are largely arbitrary (within a reasonable range). The boundaries in the $\mathrm{X}$-direction are prescribed below,

$X_{\min }=0.0, X_{\max }=5.0 \mathrm{~cm}$; 


\subsection{Solution}

The simplest CJ theory for piston problems, such as the Mader Problem, is briefly discussed in the Theory Discussion section below. In this section we just present the solution corresponding to our specific problem description.

In the Mader Problem, there is no explicit reaction chemistry, the reaction zone has zero length and the reaction energy has a fixed value. The $\mathrm{CJ}$ detonation speed is given as a parameter, along with the reaction enthalpy (chemical energy released), and the initial state ahead of the burn front. Given these parameters and the assumptions of the model, all of the remaining quantities can be computed analytically. The Hugoniot and CJ state are easly found from the jump conditions and Rayleigh line; the Euler equations yield a self-similar analytic solution for the Taylor wave behind the burn front. This is the essence of the Mader Problem.

The structure of the detonation wave in the slab is divided into three regions:

1. Ahead of the shock front, the unburned gas is in a uniform initial state.

2. The rarefaction region, proceeding from the the moving shock front to a terminal point exactly half-way between the shock front and the piston. This is a consequence of the polytropic assumption with $\gamma=3$.

3. Between the piston and the rarefaction, the material is in a uniform final state.

At the final time of $6.25 \mu \mathrm{s}$, the detonation front has reached end of the slab, at $5 \mathrm{~cm}$, and the terminal point of the rarefaction will be at $2.5 \mathrm{~cm}$. The Hugoniot relations and final-state isentrope determine the $\mathrm{CJ}$ state, which is the state of the reaction products as they exit the detonation front in Region II.

Assuming a $\gamma$-law gas, with $p=(\gamma-1) \rho e$, the $\mathrm{CJ}$ state is given by

$$
\begin{array}{r}
p_{c j}=\frac{\rho_{0} D_{c j}}{(\gamma+1)} \\
\rho_{c j}=\rho_{0}\left(\frac{\gamma+1}{\gamma}\right) \\
c_{c j}=D_{c j}\left(\frac{\gamma}{\gamma+1}\right) \\
v_{c j}=\frac{D_{c j}}{\gamma+1}
\end{array}
$$

The specific reaction enthalpy, $q$, and the postion of the detonation front at time, $t$, are given by,

$$
\begin{gathered}
q=\frac{D_{C J}^{2}}{2\left(\gamma^{2}-1\right)} \\
x_{d e t}=D_{c j} t
\end{gathered}
$$

In Region I, the material is in the constant initial state given above, but, is assumed moving in the frame of the piston in the -x direction with speed, $v_{0}=-v_{\text {piston }}$, where $v_{\text {piston }}$ is the speed of the piston in the lab frame. Thus, the initial state in Region I can be chosen to have the following simple form, consistent with the imposed Hugoniot, detonation speed and chemical energy.

$$
\begin{array}{r}
v=-v_{\text {piston }} \\
p=0.0 \\
\rho=1.857 \\
c=0
\end{array}
$$

The rarefaction fan in Region II consists of the set of characteristics, $v+c=x / t$. The flow is self-similar here, determined only by the ratio, $x / t$. The characteristics are bounded between $x / t=D_{c j}$, at the detonation front and, $x / t=D_{c j} / 2$, at the tail (for the polytropic case). So, the transition point between tail of the the rarefaction fan and 
the final state at any time is given by $x_{t a i l}=(1 / 2) D_{c j} t$. Then, the self-similar solution for the Taylor wave in Region II is found to be,

$$
\begin{array}{r}
v=\frac{\left(2(x / t)-D_{c j}\right)}{(\gamma+1)} \\
p / p_{c j}=\left[1+\frac{(\gamma-1)\left(v-v_{c j}\right)}{2 c_{c j}}\right]^{2 \gamma /(\gamma-1)} \\
\rho=\rho_{c j}\left(p / p_{c j}\right)^{1 / \gamma} \\
c=c_{c j}\left(p / p_{c j}\right)^{(\gamma-1) / 2 \gamma}
\end{array}
$$

The constant final state in Region III, equal to the state at the tail of the rarefaction. Thus we have,

$$
\begin{array}{r}
v=0 \\
p / p_{c j}=\left[1-\frac{v_{c j}(\gamma-1)}{2 c_{c j}}\right]^{2 \gamma /(\gamma-1)} \\
\rho=\rho_{c j}\left(p / p_{c j}\right)^{1 / \gamma} \\
c=c_{c j}\left(p / p_{c j}\right)^{(\gamma-1) / 2 \gamma}
\end{array}
$$

To obtain the solutions above in the lab frame, simply subtract $v_{\text {piston }}$ from all of the velocities, e.g.,

$$
v \rightarrow\left(v-v_{\text {piston }}\right)
$$

You will also need to subtract the initial postion of the detonation front from all of the $x$ quantities above if you started the detonation from some postion other than the initial position of the piston.

\subsection{Output}

In ASCII comma- or space-delimited format, ${ }^{1}$ to include:

1. Values of pressure density, pressure, material speed as a function of position at $t_{0}$ and $t_{\text {fin }}$.

2. Entire mesh total pressure density and internal energy as a function of time.

\subsection{Comparison Domain}

$X \in[0,5]$

\subsection{Theory Discussion}

The Mader Problem is based on the simplest HE detonation theory, as outlined in section 2A of Fickett and Davis [Fickett]. This theory is based on the following assumptions:

1. One-dimensional flow in a simple polytropic gas expansion.

2. The planar detonation front is a jump discontinuity or shock in which the thermodynamic path is the Rayleigh line connecting the initial and final states, as determined by the shock Hugoniot of the unreacted HE, modified by the addition of the full chemical reaction enthalpy.

\footnotetext{
${ }^{1}$ Sample output available upon request.
} 
3. The chemical reaction is assumed to burn to completion instantaneously, so the reaction products are emerge, in equilibrium, from the detonation front.

4. The detonation front motion is assumed to to be steady, so the the state of the material emerging from the shock front is time-independent.

In the simple piston detonation problem, the fuel is assumed to burn instantaneously and completely at the detonation front, which may be treated as idealized shock front, thus the material in the trailing rarefaction is assumed to consist entirely of reaction products. Therefore, the reaction enthalpy - the total heat produced by burning the fuel - is simply added to the energy Hugoniot. Using this to derive the total Hugoinot from the conservation laws, one finds that the slope of the P-V Hugoniot, which is proportional to $D_{c j}^{2}$, is only changed by the linear addition of this chemical energy. Equating the expression for the slope of the P-V Hugoniot with the slope of the Rayleigh line one finds that, $(\partial E / \partial v)_{\mathcal{H}}=-p$, on the Hugoniot. But, this relation also obtains on the isentrope of the reaction products, i.e., $(\partial E / \partial v)_{\mathcal{S}}=-p$. Therefore, the CJ state is a special point where the slope is the same for the Rayleigh line, the Hugoniot and the reaction product isentrope. This slope is proportional to $D_{c j}^{2}$. This implies that $D_{c j}$ is the stable propagation speed for the detonation wave: If the detonation wave propogates a bit faster than $D_{c j}$, the reaction products will be moving away from the detonation front faster than the local sound speed, so pressure disturbances due to the energy released from the reaction will not be able to support the shock wave. Therefore, the detonation wave should slow down until disturbances from behind "catch up". On the other hand, if the detonation wave travels a bit slower than $D_{c j}$, the sound speed for the reaction products will be greater the detonation speed, so pressure disturbances from the reaction products will overtake it, increasing the shock strength - the pressure and shock speed - until it reaches $D_{C J}$, at which point $u_{c j}+c_{c j}=D_{c j}$, i.e, the detonation is exactly sonic in the frame moving with the detonation front.

\subsection{References}




\subsection{Description}

From the conceptual description in [Kamm] as informed by [Blake]:

The Blake problem concerns the spherically symmetric propagation of radial, longitudinal waves from a cavity of radius, $a$, in a homogeneous, isotropic, linear elastic whole space, whose surface is loaded by a time-dependent normal traction or pressure. When the pressure history, $p(t)$, is a step function or other rapid increase in time followed by a suitable decay, the solution has application in modeling the behavior of an embedded explosive energy source. We consider the simplest case in which the applied pressure history is a step function at $t=0$.

This problem tests the hydrocode's ability to calculate outgoing, spherically divergent elastic wave propagation in the absence of boundary reflections. This includes the code's ability to accurately simulate the wave propagation with minimal use of artificial viscosity and to conserve energy well as the physics is dissipation free.

This is a closed-form, analytical solution (type 2 of [Oberkampf]).

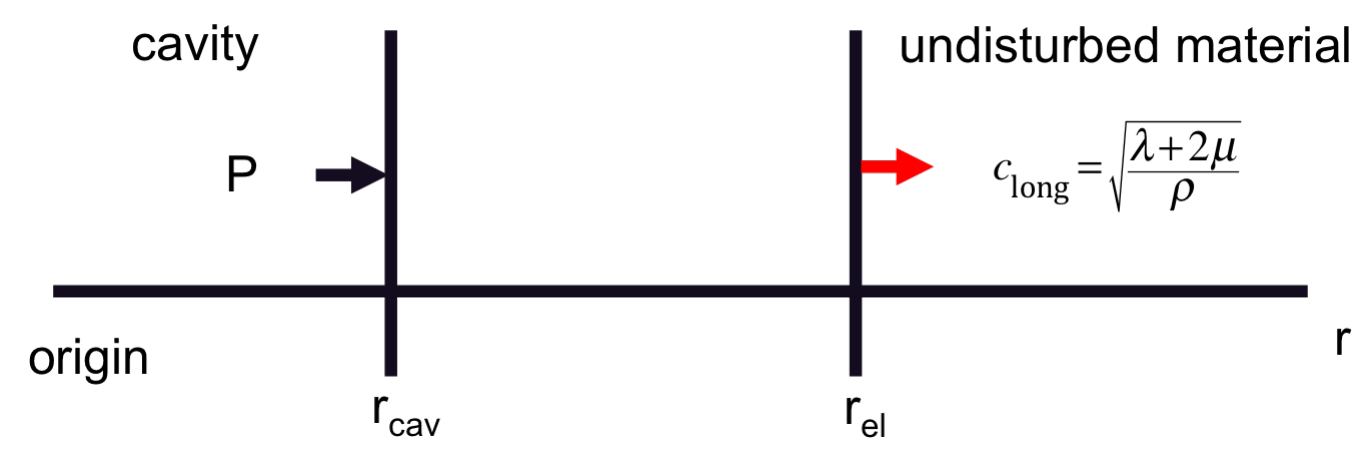

Fig. 16.1: Schematic of the physical arrangement for the Blake problem. 


\subsection{Problem Parameters}

Table 16.1: Parameters for the Blake problem.

\begin{tabular}{|l|l|}
\hline Parameter Name & Default Value \\
\hline Reference density & $\rho=3.0 \mathrm{~kg} / \mathrm{m}^{3}$ \\
\hline Shear modulus & $\mu=25.0^{*} 10^{9} \mathrm{~Pa}$ \\
\hline Poisson ratio & $\nu=0.25$ \\
\hline Cavity radius $\left(\mathrm{r}_{\text {cav }}\right)$ & $\mathrm{r}_{\text {cav }}=0.1 \mathrm{~m}$ \\
\hline Max. mesh radius & $\mathrm{r}_{\text {outer }}=1.2 \mathrm{~m}$ \\
\hline Pressure scale & $\mathrm{P}_{0}=1.0^{*} 10^{6} \mathrm{~Pa}$ \\
\hline Final (snapshot) time & $\mathrm{t}_{\text {snap }}=1.6^{*} 10^{-4} \mathrm{~s}$ \\
\hline
\end{tabular}

\subsection{Linear Elastic Parameters}

There are six parameters which are commonly used to characterize an isotropic, linear-elastic solid.

\begin{tabular}{|l|c|}
\hline Parameter Name & Symbol \\
\hline First Lame Modulus & $\lambda$ \\
\hline Shear Modulus (Second Lame Modulus) & $G$ \\
\hline Young's Modulus & $E$ \\
\hline Poisson's Ratio & $\nu$ \\
\hline Bulk Modulus & $K$ \\
\hline Longitudinal Modulus & $M$ \\
\hline
\end{tabular}

The Blake solver accepts any two of these to create a solver instance for a particular elastic material. See the solver documentation for further detail.

\subsection{Initial Conditions}

Undistorted, unstressed, zero-velocity isotropic linear-elastic material.

\subsection{Boundary Conditions}

A time-constant pressure $\left(\mathrm{p}_{0}\right)$ is applied to the cavity surface at $t=0$. The computational domain outer boundary is chosen such that the leading edge of the wave structure is well within the domain at $t_{\text {snap }}, r_{\text {max }}=0.9 m<r_{\text {outer }}$.

\subsection{Mesh (1D spherical)}

Domain:

$r_{\min }=r_{\text {cav }}, r_{\max }=r_{\text {outer }}$

Mesh Resolutions:

$N_{\text {zones }}=44,88,176,352,704, \Delta_{r}=0.025,0.0125,0.00625,0.003125,0.0015625$ 


\subsection{Output}

In ASCII comma- or space-delimited format, to include:

Values of density and pressure, radial, hoop and volumetric stress, strain and stress deviators, as a function of position at a user-specified $t_{\mathrm{snap}}$, not greater than the final time indicated in the table.

\subsection{Results (default parameters) at $t_{\text {snap }}=1.6 * 10^{-4} \mathrm{~s}$}
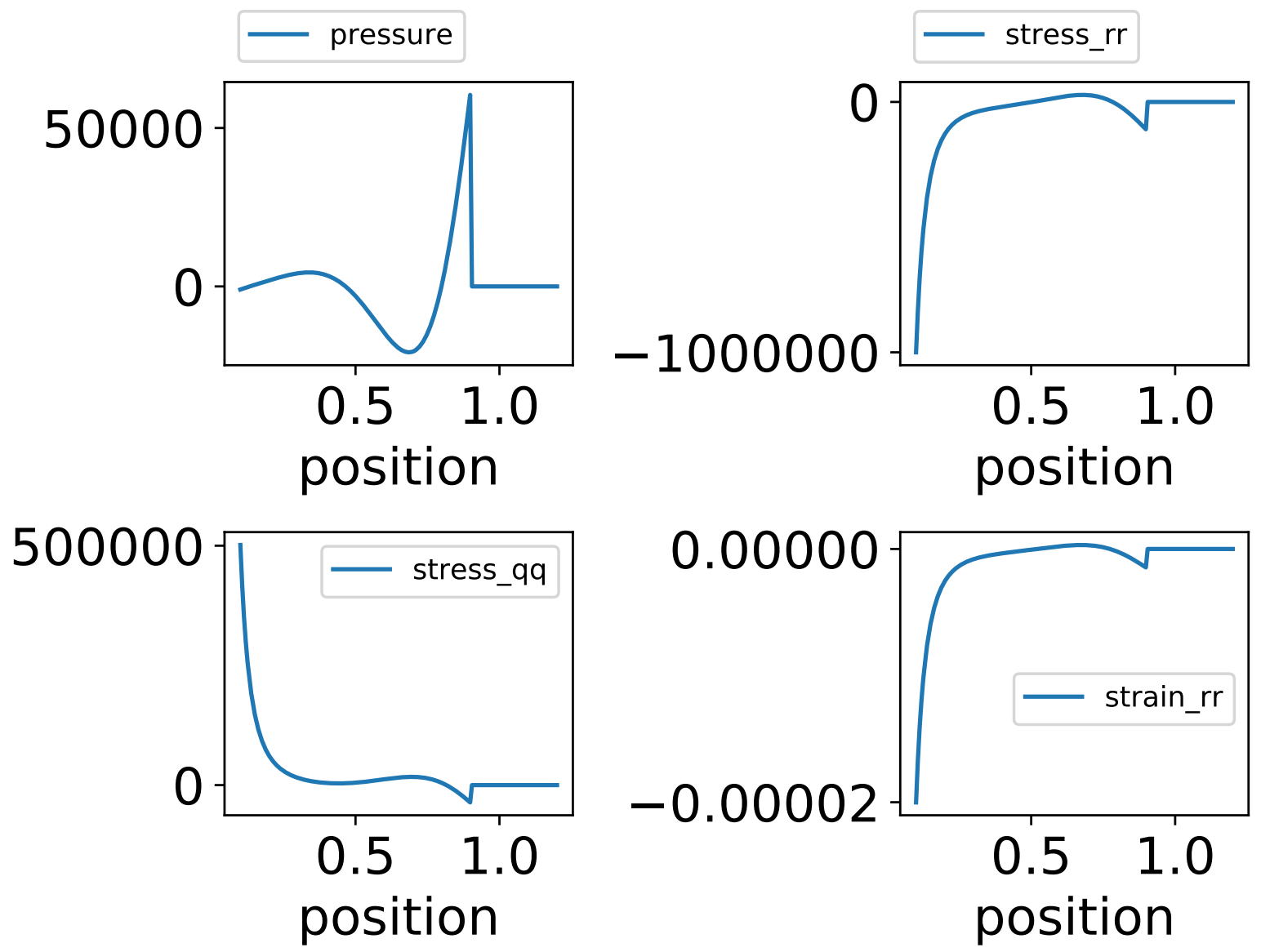


\subsection{Description}

From the conceptual description in [Kamm]:

Hunter's Problem consists of an infinite, uniform, elastic-perfectly-plastic (EPP) medium, containing a spherical inclusion of radius $a$ about the origin. At initial time $(\mathrm{t}=0)$, the boundary of the inclusion $(\mathrm{r}=\mathrm{a})$ is subject to a specified, time-dependent driving pressure $\mathrm{P}$; this driving pressure has a complicated but closed form, given in [Hunter], which can be evaluated numerically and used to drive a hydrocode simulation. The specified driving pressure generates elastic-plastic waves such that the boundary between elastic and plastic deformation moves outward from the cavity wall with constant radial velocity. This problem is, in some sense, a generalized EPP analogue of the Blake problem [Blake].

This problem tests outgoing, spherically divergent elastic-plastic wave propagation in the absence of boundary reflections. This includes the interaction between elastic and plastic states at the elastic-plastic boundary, as well as the dissipation of energy through plastic work.

This is a closed-form, analytical solution (type 2 of [Oberkampf]).

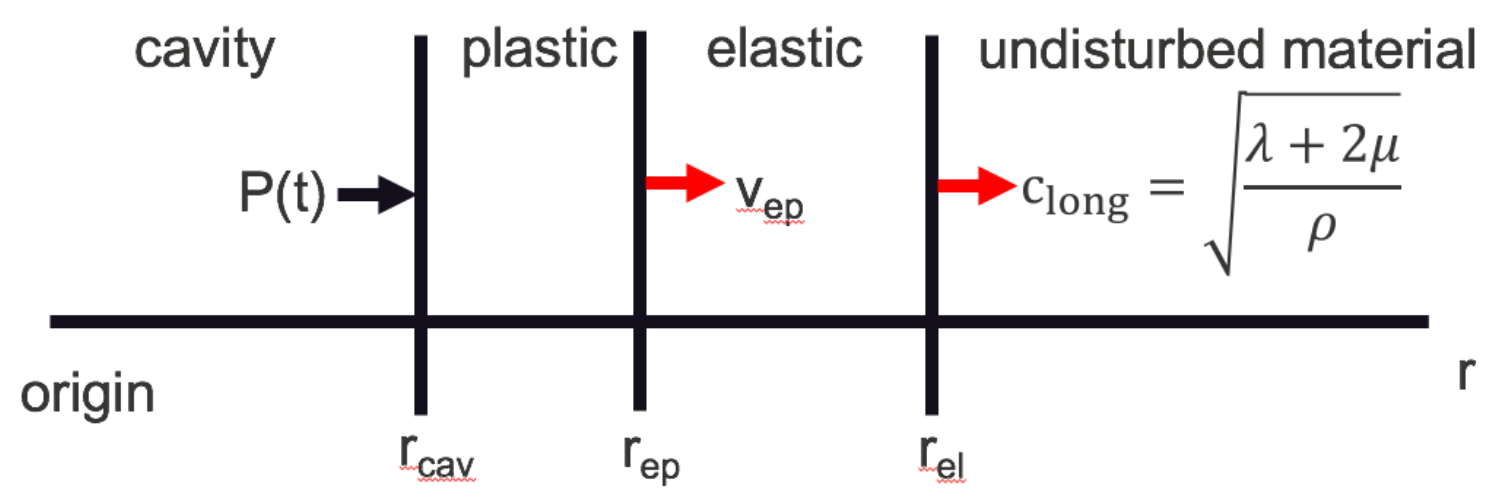

Fig. 17.1: Schematic of physical situation for Hunter's problem. 


\subsection{Problem Parameters}

Table 17.1: Parameters for the Hunter problem.

\begin{tabular}{|l|l|}
\hline Parameter type & Value \\
\hline Cavity boundary $\left(\mathrm{r}_{\text {cav }}\right)$ & $\mathrm{a}=100.0 \mathrm{~cm}$ \\
\hline Density & $\rho=1.0 \mathrm{~g} / \mathrm{cm}^{3}$ \\
\hline Poisson ratio & $\nu=1 / 3$ \\
\hline Bulk modulus & $\kappa=10.0^{*} 10^{9} \mathrm{dyne} / \mathrm{cm}^{2}$ \\
\hline Yield strength & $\mathrm{Y}=1.0^{1} 10^{8} \mathrm{dyne} / \mathrm{cm}^{2}$ \\
\hline Elastic-plastic interface speed & $\mathrm{c}_{\mathrm{ep}}=2.4495^{*} 10^{4} \mathrm{~cm} / \mathrm{s}$ \\
\hline Final time & $\mathrm{t}_{\text {snap }}=0.001 \mathrm{~s}$ \\
\hline Max. mesh radius & $\mathrm{r}_{\text {final }}=300.0 \mathrm{~cm}$ \\
\hline
\end{tabular}

\subsection{Initial Conditions}

Uniform, constant, zero-velocity elastic-perfectly-plastic material with a vacuum cavity.

\subsection{Boundary Conditions}

The closed form solution for the pressure applied at the inner boundary is given in [Hunter]. This is a complicated form, but is an algebraic closed form. The outer boundary is chosen such that the outgoing wave will not interact.

\subsection{Mesh}

$R_{\min }=100.0, R_{\max }=300.0 \mathrm{~cm}$; in $1 \mathrm{D}$

Mesh Resolutions:

1D spherical: $N_{R}=100,200,400,800,1600 ; \Delta_{R}=2.0,1.0,0.5,0.25,0.125$

\subsection{Output}

In ASCII comma- or space-delimited format, to include:

1. Values of pressure, radial stress, hoop stress, and deviators for the stresses as a function of position at $t_{\text {snap }}$.

2. Entire mesh total energy as a function of time.

\subsection{Comparison Domain}

1D: $R \in[0,3.0]$ where the position has been scaled by a.

\footnotetext{
${ }^{1}$ Note that this value is $1 / 10$ th the value listed in $[\mathrm{Kamm}]$. This value was chosen due to the assumption of small strain in the original derivation. Large yield strength results in overdriven solutions.
} 


\subsection{Results at $t_{\text {snap }}=0.001 \mathbf{s}$}

All quantities have been scaled as in [Hunter] with pressure and stress divided by the yield strength. Strain is divided by the quantity

$$
W=\frac{\text { yield stress }}{2 * \text { second Lame constant }}
$$




\section{BIBLIOGRAPHY}

[Fickett] Fickett, W., and Davis, W.C., Detonation, (UC Berkeley, 1979)

[Timmes] Francis X. Timmes, Galen Gisler, George M. Hrbek, Automated Analyses of the Tri-Lab Verification Test Suite on Uniform and Adaptive Grids for Code Project A, LA-UR-05-6865

[Kirkpatrick] Kirkpatrick, R., Wingate, C., Kamm, J., HE Burn Test Problem, X-3-19U, 2004

[Kamm] Kamm, et al.,’Enhanced Verification Test Suite for Physics Simulation Codes”, LA-14379

[Blake] Blake, Jr., F. G. "Spherical wave propagation in solid media”, J. Acous. Soc. Am. 24: 211-215 (1952).

[Coggeshall] Coggeshall, S. V., “Analytic Solutions of Hydrodynamics Equations”, Phys. Fluids A 3 (5), May 1991

[Doebling] S. Doebling, "The Escape of High Explosive Products: An Exact-Solution Problem for Verification of Hydrodynamics Codes," Journal of Verification, Validation and Uncertainty Quantification, 1(4), 2016, http:// verification.asmedigitalcollection.asme.org/article. aspx?articleid=2575765

[Dykema] P. Dykema, S. Brandon, J. Bolstad, T. Woods, and R. Klein, Level 1 V.\&V. Test Problem 10: Escape of High Explosive Products, LLNL Report, UCRL-ID-150418, Lawrence Livermore National Laboratory (2002).

[Dobratz] Dobratz, B.M. and Crawford, P.C., "LLNL Explosives Handbook: Properties of Chemical Explosives and Explosive Simulants", LLNL Report, UCRL-52997, Jan 1985.

[Fickett] W. Fickett and C. Rivard, Test Problems for Hydrocodes. LASL Report, LA-5479, Los Alamos Scientific Laboratory (1974, Rev 1981).

[Guderley] Guderley, G. (1942). "Starke kugelige und zylindrische Verdichtungsstoße in der Nahe des Kugelmittelpunktes bzw. der Zylinderachse", Luftfahrtforschung, 19, 302-312.

[Hendon] Hendon, R and Ramsey, S., "Conduction Invariance in Similarity Solutions for Compressible Flow Code Verification", LA-UR-15-20020 (2015)

[Hunter] Hunter, S. C. "The Propagation of Spherically Symmetric Disturbances in Ideally Plastic Materials". In "Proceedings of the Conference on Properties of Materials at High Rates of Strain, London 1957, pp. 147-155 (1957).

[Kamm] Kamm, et al.,”Enhanced Verification Test Suite for Physics Simulation Codes”, LA-14379 (2008)

[Kenamond] Kenamond, M. A., “HE Burn Table Verification Problems”, LA-UR 11-03096, 2011.

[Kidder] Kidder, R. E., "Theory of Homogeneous Isentropic Compression and its Application to Laser Fusion", Nuclear Fusion 14 (1974).

[Lee] Lee, B.J., Toro, E.F., Castro, C.E., Nikoforakis, N., “Adaptive Osher-Type Scheme for Euler Equations with Highly Nonlinear Equations of State”, J. Comput. Phys. 246:165-182 (2013)

[Molvig] Molvig, K., et al., "Nonlinear Structure of the Diffusing Gas-Metal Interface in a Thermonuclear Plasma", Phys. Rev. Lett. 113, 145001 (2014). 
[Noh] W. Noh, "Errors for Calculations of Strong Shocks Using an Artificial Viscosity and an Artificial Heat Flux," J.Comput. Phys. 72:78-120 (1987).

[Oberkampf] Oberkampf, W. L. and Trucano, T. G. "Verification and Validation Benchmarks", Sandia National Laboratories report SAND2007-0853 (2007).

[Ramsey] Ramsey, S. D., et al., "A Class of Self-Similar Hydrodynamics Test Problems”, NECDC 2010 Proceedings, LA-UR-10-08184 (2010).

[Shyue] Shyue, K.-M. "A Fluid-Mixture Type Algorithm for Compressible Multicomponent Flow with MieGrüneissen Equation of State", J. Comput. Phys 171:688-707 (2001) 